\begin{tabular}{|c|l|}
\hline Title & Energy Filtered X-Ray Photoemission Electron Microscopy \\
\hline Author(s) & A sakura, Kiyotaka; Niimi, Hironobu; Kato, Makoto \\
\hline Citation & $\begin{array}{l}\text { Advances in imaging and electron physics, 162(2), 1-209 } \\
\text { https://doi.org/10.1016/S1076-5670(10)62001-0 }\end{array}$ \\
\hline Issue Date & 2010 \\
\hline Doc URL & http://hdl.handle.net/2115/51690 \\
\hline Type & article (author version) \\
\hline File Information & S90569-1A 3a2-1.pdf \\
\hline
\end{tabular}

Instructions for use 


\title{
Energy Filtered X-ray Photoemission electron microscopy(EXPEEM)
}

\author{
Kiyotaka Asakura $^{1}$, Hironobu Niimi ${ }^{1,2}$, Makoto Kato ${ }^{2}$ \\ ${ }^{1}$ Catalysis Research Center, Hokkaido University, Kita 21 Sapporo 001-0021, Japan
}

JEOL Ltd., 3-1-2 Musashino, Akishima, Tokyo 196-8558, Japan

${ }^{2}$ Photoemission electron microscopy (PEEM) and energy filtered X-ray PEEM (EXPEEM) are

reviewed. PEEM enables the in situ observation of surface chemical phenomena resolved in space and time. In order to achieve surface chemical imaging, excitation at the core level is preferable and a combination of PEEM and X-ray photoelectron spectroscopy is necessary which has been realized by the EXPEEM. We review in particular an EXPEEM installed with a Wien filter energy analyzer are reviewed. Examples of the practical application of PEEM and EXPEEM to surface chemistry are presented.

\section{Abstract}

This chapter reviews photoemission electron microscopy(PEEM) and energy filtered X-ray PEEM (EXPEEM) that provides us the chemical mapping of the surface. We describe the history and the principle of PEEM and EXPEEM. We focus on the Wien filter type energy analyzer with $3^{\text {rd }}$ aberration corrected. In situ observation of surface reactions and chemical mapping of soft materials are discussed as exmples of the PEEM and EXPEEM applications to 
chemistry.

1. Introduction

Photoemission electron microscopy (PEEM) is a method to investigate chemical phenomena at the surface with spatial and temporal resolution. In PEEM, photoelectrons excited by light are collected by a cathode lens (or a magnetic objective lens) followed by intermediate and projection lenses used to magnify and project the surface image onto a screen. The contrast of the image is formed by the amount of emitted photoelectrons. Figure 1 shows a PEEM image of spatiotemporal patterns of $\mathrm{CO}$ and $\mathrm{O}_{2}$ adsorbed on a $\mathrm{Pt}(110)$ surface during $\mathrm{CO}$ oxidation. ${ }^{1}$ The surface is illuminated by a UV lamp and photoelectrons are ejected from the surface, depending on the local work function. The work function of $\mathrm{O}_{2}$ is larger than $\mathrm{CO}$ and the amount of electrons coming from the $\mathrm{O}_{2}$-adsorbed surface becomes smaller; therefore, the dark and bright regions correspond to the $\mathrm{O}_{2}$ and $\mathrm{CO}$ adsorbed domains, respectively. PEEM had revealed that surface reactions are inhomogenous, even on a single crystal and in situ surface microscopy is necessary since the pattern is formed under reaction conditions.

The PEEM image in Fig. 1 shows the distribution of $\mathrm{O}_{2}$ and $\mathrm{CO}$ that can be obtained only by their different work functions when they are adsorbed on a surface. Therefore, PEEM can not 
be applied to complex systems where the work functions of the reaction components are not so easily distinguished. However, PEEM has the following advantages: 1) less specimen damage than conventional electron microscopy, and 2) versatility to provide physical and chemical information by the selection of excitation photon sources. ${ }^{2,3}$ In contrast to transmission electron microscopy (TEM) or scanning electron microscopy (SEM), PEEM requires a photon source that has a small interaction with matter, resulting in minimal sample damage. The second advantage is related to the photoemission mechanism of PEEM. Although the amount of photoelectrons excited by a simple UV light source is simply related to the work function of the surface, ${ }^{3}$ the number of excited photoelectrons depends on the local magnetism of the surface when circularly or linearly polarized light is used. ${ }^{4,5}$ X-rays excite the core electrons, which contain chemical information. Synchrotron radiation ${ }^{6}$ is an energy-tunable X-ray source, which can be used to obtain chemically-sensitive PEEM images when the X-ray energy is set near the absorption edge energy. On the other hand, kinetic energy analysis of the photoelectrons is necessary to obtain chemically sensitive PEEM images for a constant photon energy source such as conventional Bremsstrahlung X-rays. PEEM combined with photoelectron energy analysis is referred to as an energy filtered X-ray PEEM (EXPEEM). ${ }^{7-13}$ One challenging aspect is the weak photoelectron peak signal arising from the core electrons.

In this paper, we first review the history of PEEM and EXPEEM. The PEEM and 
EXPEEM instrumentation are then described, which includes the photon source, although the electron lens system is briefly covered. We present various methods of photoelectron energy analyzer (EA). An EXPEEM with a Wien filter type EA is a main focus of this paper. The multipole Wien filter EA is suitable for application to EXPEEM because of its collinear optical axis. Finally some recent applications of PEEM and EXPEEM are given, with respect to chemical problems.,

\section{History ${ }^{14}$}

\subsection{Early Development}

PEEM has a long history starting from 1930s, at approximately the same time as the invention of TEM. ${ }^{15}$ Bruche reported photoelectron images obtained using the simple lens system shown in Fig. $2^{16}$

Bruche obtained a $2 \times$ magnification image of a Zn plate. The electrons coming from the sample were accelerated by an electric field between the sample and the objective lens of microscope (R). The electrons were focused on the screen using the magnetic field produced by the magnet (M). Pohl et al. applied PEEM and thermionic emission electron microscopy (TEEM) to the metallurgy and observed crystal grains of a platinum surface. ${ }^{17}$ Although emission electron microscopy was investigated by many pioneers at that time, ${ }^{14}$ wide utilization of PEEM required the development of ultrahigh vacuum (UHV) and surface cleaning techniques, 
because PEEM is very sensitive to the surface state.

\subsection{Development after 1980}

Surface science techniques using single crystals under UHV condition were established in the 1970s. ${ }^{18}$ In the 1980s, Bauer ${ }^{19}$ and Ichinokawa ${ }^{20}$ developed UHV-compatible modern emission electron microscopy with electrons as the excitation source, referred to as low energy electron microscopy (LEEM ). LEEM employs electron diffraction at low kinetic energies as a contrast mechanism; therefore, beam separator electrode (BSE)is necessary to separate the primary and emitted electrons in the LEEM. PEEM can employ the same system as LEEM, only with the excitation source changed to UV light. A more compact PEEM with electrostatic lenses was developed by Engel and coworkers, which was used for in situ observation of surface phenomena as shown in Fig. 3a.,3,21,22 In the 1990s, synchrotron radiation became an everyday tool and was used as a powerful excitation source for PEEM. The tunability of the photon energy, X-ray pulse and polarization (circular or linear) of synchrotron radiation provides surface mapping according to chemical and physical information, such as elemental distribution, molecular orientation, and magnetic domain. ${ }^{23}$ The pulse properties enable time-resolved measurements. Vogel et al. applied pump-probe X-ray PEEM to the measurement of a permalloy layer composed of a $\mathrm{Ni}_{80} \mathrm{Fe}_{20}(5 \mathrm{~nm}) / \mathrm{Cu}(10$ $\mathrm{nm}$ )/Co (5 nm) trilayer deposited on Si(111). ${ }^{24}$ Short magnetic field pulse (pump) induces the 
nucleation and subsequent growth of reversed magnetic domains in the permalloy.

\subsection{EXPEEM}

The first example of EXPEEM can be found in photoelectron spectromicroscopy (PESM) pioneered in 1980 by Beamson, Porter and Turner. ${ }^{25,26}$ They used a solenoid coil placed behind the sample that produced a divergent magnetic field, as shown in Fig. 3b.

The ejected photoelectron, with a kinetic energy more than $100 \mathrm{eV}$, can assume a helical motion around the divergent magnetic field while its energy and orbital moment are conserved.

The areal magnification is given as $\mathrm{B}(2) / \mathrm{B}(1)$, where $\mathrm{B}(2)$ and $\mathrm{B}(1)$ are the magnetic fields at the object and the image planes, respectively. ${ }^{27}$ Energy analyses were carried out in the image band pass filter. $^{28}$ PESM did not employ an electron lens. Tonner et al. combined PEEM and X-ray photoelectron spectroscopy (XPS), ${ }^{29,30}$ to produce the system shown schematically in Fig. 4 where PEEM shown in Fig.3a is divided into two parts : One part is input and intermediate lenses and the other is projection lens to magnify and project the surface images. Between them a hemispherical type energy analyzer is inserted to select the photoelectron peaks.

\section{Principle and Experimental Setup of PEEM and EXPEEM}

\subsection{Photoemission and Light Source}

PEEM contrast can be formed by differences in the amount of ejected electrons on a surface.

When UV light is used, the amount of photoelectrons, $I$, is proportional to the work function, 
$\chi$.

$I \propto(\chi-h v)^{2}$

(1)

The work functions of metals are usually several eV (less than $10 \mathrm{eV}$ ); ${ }^{31}$ therefore, $\mathrm{Hg}$ and $\mathrm{D}_{2}$

lamps are often used. $\quad \mathrm{A} \mathrm{D}_{2}$ lamp has a peak at $6 \mathrm{eV}$ that can excite electrons from a Pt surface

with a work function of around $5.7 \mathrm{eV}$, but not from an $\mathrm{O}_{2}$ covered Pt surface.

Fig. 5 shows PEEM images of $10 \mu \mathrm{m}$ sized Au islands on a Ta substrate excited by $\mathrm{D}_{2}$ lamp ${ }^{32}$ during heat treatment. The Ta substrate is covered with a native Ta oxide film at $300 \mathrm{~K}$ and Ta oxide has a larger work function, so that the Au region is brighter than the Ta substrate.

When the sample is heated to $723 \mathrm{~K}$, the Ta oxide film is removed and the clean Ta region is exposed. The work function of metallic Ta is smaller than that of $\mathrm{Au}$, so that the contrast of the Au and Ta regions are reversed, as shown in Fig. 5b. At $773 \mathrm{~K} \mathrm{Au}$ and Ta alloy was formed in the Au-pre-corever region shown in Fig. 5c which revealed unique behavior to $\mathrm{H}_{2}$ adsorption and absorption discussed later. At $1100 \mathrm{~K} \mathrm{Au}$ is evaporated from the surface and the contrast becomes weak, as shown in Fig. 5d.

Therefore, the work function can be modified according to the surface composition. A Cs covered metal or semiconductor has a lower work function than the bare surface without Cs. Nakagawa et al. controlled the work function of a $\mathrm{Ni}$ thin layer surface on $\mathrm{Cu}(001)$ by a continuous Cs deposition ${ }^{33,34}$ and investigated electronic structure and magnetic properties of 
the Ni thin layer using PEEM with a photon source of constant energy that is less than the work function of the surface without Cs. Cs is assumed to only decrease the work function and not to affect the bulk electronic structure. The work function continuously decreases by gradually increasing the amount of Cs. When the work function becomes less than the photon energy, photoemission starts to occur. Nakagawa et al. obtained an enhanced magnetic circular dichroism (MCD) PEEM image at the threshold energy of the photoemission.

EXPEEM can provide direct chemical imaging. When X-rays are used, the core electron can be excited, of which the energy reflects the elemental and chemical states. Since the amount of photoelectrons emitted from core electrons is much smaller than that for UV excitation, an intense X-ray source is required. In order to obtain a high power X-ray, a rotatory anode in combination with an X-ray focusing monochromator is used. Fig. 6 shows a rotatory anode X-ray source with a $400 \mathrm{~W}$ Al target. The X-rays are dispersed over a wide angle and the flux is small per area (less than $1 \mathrm{~mm}^{2}$ ). Thus the X-rays should be focused on the sample. Since the refraction index of matters is almost unity, there were no X-ray lens in a general sense and a focusing mirror requires grazing incidence which decreases the acceptance angle. The Bragg diffraction is used to focus the X-rays onto the small area of the sample. Multilayer troidal monochromator composed of alternating layers of Mo and C is set between the X-ray source and the sample so that the X-rays are effectively condensed to 
$300 \times 200 \mu \mathrm{m}^{2}$ region with the photon density of $10^{11}$ photons $/ \mathrm{mm}^{2} / \mathrm{s}$. In addition to focusing the X-rays, the monochromator can select the desired characteristic X-ray peak to reduce the background or satellite peaks produced by X-rays with other energies.

Synchrotron radiation is the most suitable photon source for PEEM and EXPEEM. Synchrotron radiation is emitted from electrons traveling near light speed $v \approx c$, through a magnetic field. Synchrotron radiation has the following features:

1) a collimated beam with a radiation emission angle $\psi=1 / \gamma$ in the traveling direction of the electron,

2) a pulsed light with a pulse width of $\frac{1}{\omega \gamma^{3}}$ and a pulse interval of $\frac{2 \pi}{\omega}$,

3) white light or a wide range of the energy spectrum with cut off energy $\varpi_{c}=\frac{3}{2 \gamma^{3} \varpi}$,

4) linearly polarized or circularly polarized light,

where $\gamma=1 / \sqrt{1-(\mathrm{v} / \mathrm{c})^{2}}$ and $\omega$ is the angular speed of an electron.

The photon energy can be selected using a crystal or grating monochromator. ${ }^{35}$ The tunability of the X-ray energy enables chemically sensitive PEEM. When the X-rays are used with energy slightly higher than the binding energy of the core electrons in a specific element, the region with that element becomes brighter. Fig. 7 shows Au islands on a Ta substrate. When the sample is illuminated with X-rays at $2195 \mathrm{eV}$, which is slightly higher than Ta $3 \mathrm{p}_{3 / 2}$, then the Ta region appears brighter. When the X-ray energy is tuned to the $\mathrm{Au} \mathrm{M}_{\mathrm{V}}$ edge or 
$3 d_{5 / 2}$ binding energy $(2240 \mathrm{eV})$, the contrast becomes weak, as shown in Fig. $7 \mathrm{~b}$. When the photon energy is tuned to $2290 \mathrm{eV}$, which is just higher than the $\mathrm{Au} \mathrm{M}_{\mathrm{V}}$ edge or $3 \mathrm{~d}_{5 / 2}$ binding energy ( $2240 \mathrm{eV})$, the Au regions become much brighter. The sizes of the Au domains in Figs. 7a and c are different, due to the slit effect of the Au islands. ${ }^{7}$

Unlike the normal synchrotron radiation emitted from the near light-speed electron bent at a magnetic field once as shown in Fig. 8a, undulator radiation is the light from the electron going through a series of magnets with alternative magnetic fields, shown in Fig. 8b, where the electron is bent several times and emits light at each bending. The maximum deflection angle of the electron $\psi_{0}$, is smaller than the radiation emission angle $1 / \gamma=\sqrt{1-\left(\frac{v}{c}\right)^{2}}$, so that the electron emission is always observed. In this case, the emitted light interferes with each other and the light from the undulator radiation becomes stronger and more monochromatic. ${ }^{35}$ The feature of the undulator can be represented by $K$ (deflection parameter), which is defined as $K=\psi_{0} \cdot \frac{1}{\gamma}=0.934 B_{0}(T) \lambda_{u}(\mathrm{~cm})$, where $B_{0}$ and $\lambda_{u}$ are the maximum magnetic flux density and the periodic length of the magnet, respectively. The undulator radiation condition is a $\mathrm{K}<1$.

The wavelength and flux density of the first order undulator beam on axis are expressed as,

$$
\begin{aligned}
& \lambda_{1}=\frac{\lambda_{u}}{2 \gamma^{2}}\left[1+\frac{K^{2}}{2}\right] \\
& \frac{d^{2} P_{1}}{d \omega d \Omega} \mid=4.555 \times{ }^{4} I(m A) \gamma^{2} N^{2} G_{1}(K)\left(\text { photons } / \mathrm{s} / \mathrm{mrad}^{2} / 0.1 \%\right. \text { band width) }
\end{aligned}
$$

where $\omega$ and $\Omega$ are the photon energy and solid angle, respectively. $I$ and $N$ are the 
electron current and the number of periods, respectively. $G_{1}(K)$ is given by the Bessel functions as follows.

$$
\begin{aligned}
& G_{1}(K)=\xi^{2} K^{2}\left\{J_{0}\left(Y_{0}\right)-J_{1}\left(Y_{0}\right)\right\}^{2} \\
& Y_{0}=\xi \frac{K^{2}}{4} \\
& \xi=\frac{1}{1+\frac{K^{2}}{2}}
\end{aligned}
$$

Thus, the photon energy of the undulator radiation can be controlled by the magnetic field strength through the $\mathrm{K}$ value. When the electron travels through a helical undulator where two crossed and overlapped magnet arrays are placed alternately, an electron takes a helical orbit, as shown in Fig. 8(c), to produce circularly polarized light. The circularly polarized light eject a specific amount of photoelectrons according to the magnetic dipole of the sample surface. The selection rule of the transition induced by circularly polarized light with its helicity $=1(-1)$ is the change in the magnetic quantum number by $1(-1)$ while spin momentum is conserved. ${ }^{36}$ The transition of $2 \mathrm{p}$ to $3 \mathrm{~d}$ absorption is now considered, which is an electric dipole allowed transition of which the transition probability is proportional to $\left|\left\langle 2 m_{d}\left|\mathbf{r}_{ \pm}\right| 1 m_{p}\right\rangle\right|^{2}=\left.\left\langle 2 m_{d}\left|\frac{x \pm i y}{\sqrt{2} r}\right| 1 m_{p}\right\rangle\right|^{2}=\mid c^{1}\left(2 m_{d},\left.1 m_{p}\right|^{2}\right.$ where $\left|c^{1}\left(2 m_{d}, 1 m_{p}\right)\right|^{2}$ is a Gaunt coefficient. ${ }^{37}$ The circular polarization is expressed by $\mathbf{r}_{ \pm}$, where $\mathbf{r}_{+}$has helicity $=1$ and $\mathbf{r}_{-}$has helicity $=-1$. 


$$
\begin{aligned}
& |c(2,1)|^{2}=6,|c(1,0)|^{2}=3,|c(0,-1)|^{2}=1 \\
& |c(-2,-1)|^{2}=6,|c(-1,0)|^{2}=3,|c(0,1)|^{2}=1
\end{aligned} \text { for } \begin{gathered}
\text { helicity =1 } \\
\text { helicity =-1 }
\end{gathered} \text {, respectively. }
$$

There are two absorption edges related to the $2 p$ to $3 d$ transition, called $L_{3}$ and $L_{2}$, where the final $2 \mathrm{p}$ hole is located at the $2 \mathrm{p}_{3 / 2}$ and $2 \mathrm{p}_{1 / 2}$ states, respectively.

$2 p_{3 / 2}$ state has four degenerated states

$$
\mid j m_{j}>=\left\{\begin{array}{c}
\left|\frac{3}{2}, \frac{3}{2}\right\rangle \propto Y_{1}^{1} \alpha \\
\left|\frac{3}{2}, \frac{1}{2}\right\rangle \propto \sqrt{\frac{2}{3}} Y_{1}^{0} \alpha+\sqrt{\frac{1}{3}} Y_{1}^{1} \beta \\
\left|\frac{3}{2},-\frac{1}{2}\right\rangle \propto \sqrt{\frac{1}{3}} Y_{1}^{-1} \alpha+\sqrt{\frac{2}{3}} Y_{1}^{0} \beta \\
\left|\frac{3}{2},-\frac{3}{2}\right\rangle \propto Y_{1}^{-1} \beta
\end{array}\right.
$$

$2 \mathrm{p}_{1 / 2}$ has two following states

$$
\mid j m_{j}>=\left\{\begin{array}{l}
\left|\frac{1}{2}, \frac{1}{2}\right\rangle \propto-\sqrt{\frac{1}{3}} Y_{1}^{0} \alpha+\sqrt{\frac{2}{3}} Y_{1}^{1} \beta \\
\left|\frac{1}{2},-\frac{1}{2}\right\rangle \propto-\sqrt{\frac{2}{3}} Y_{1}^{-1} \alpha+\sqrt{\frac{1}{3}} Y_{1}^{0} \beta
\end{array}\right.
$$

When the magnetic material is placed in a magnetic field, the final state $\mathrm{d}$ orbitals are mainly split according to the spin and is slightly affected by the magnetic quantum number. Thus, the final state for both spin directions can be classified as the state $\mid 2, m_{d}>$ state.

For $2 \mathrm{p}_{3 / 2} \quad\left(\mathrm{~L}_{3}\right.$ edge $)$ and helicity $=1$, the transition probability ratios for each $\mid 2, m_{d}>$ with 
helicity $=1$ are expressed as

$$
\begin{aligned}
& \left|\left\langle 22\left|\mathbf{r}_{+}\right| 3 / 2,3 / 2\right\rangle\right|^{2}=18 \\
& \left|\left\langle 21\left|\mathbf{r}_{+}\right| 3 / 2,1 / 2\right\rangle\right|^{2}=6 \\
& \left|\left\langle 20\left|\mathbf{r}_{+}\right| 3 / 2,-1 / 2\right\rangle\right|^{2}=1
\end{aligned}
$$

For light with opposite helicity,

$$
\begin{aligned}
& \left.\left\langle 20\left|\mathbf{r}_{-}\right| 3 / 2,3 / 2\right\rangle\right|^{2}=3 \\
& \left|\left\langle 2-1\left|\mathbf{r}_{-}\right| 3 / 2,1 / 2\right\rangle\right|^{2}=6 \\
& \left\langle\left.\left\langle 2-2\left|\mathbf{r}_{-}\right| 3 / 2,-3 / 2\right\rangle\right|^{2}=6\right.
\end{aligned}
$$

The transition probability ratios for the minor spin states of both helicities are given as

$$
\begin{aligned}
& \left|\left\langle 2,2\left|\mathbf{r}_{+}\right| 3 / 2,1 / 2\right\rangle\right|^{2}=6 \\
& \left|\left\langle 2,1\left|\mathbf{r}_{+}\right| 3 / 2,-1 / 2\right\rangle\right|^{2}=6 \\
& \left|\left\langle 2,0\left|\mathbf{r}_{+}\right| 3 / 2,-3 / 2\right\rangle\right|^{2}=3 \\
& \left|\left\langle 2,0\left|\mathbf{r}_{-}\right| 3 / 2,1 / 2\right\rangle\right|^{2}=1 \\
& \left|\left\langle 2,-1\left|\mathbf{r}_{-}\right| 3 / 2,-1 / 2\right\rangle\right|^{2}=6 \\
& \left|\left\langle 2,-2\left|\mathbf{r}_{-}\right| 3 / 2,-3 / 2\right\rangle\right|^{2}=18
\end{aligned}
$$

The empty $d$ state is written as $h_{m}$, where $m$ is the magnetic quantum number. The absorption intensity is the sum of the product of the number of empty d states and the transition probability.

$$
I_{ \pm}=\sum_{m, J_{m}} h_{m}\left|\left\langle 2, m\left|\mathbf{r}_{ \pm}\right| 3 / 2, J_{m}\right\rangle\right|^{2}
$$


intensity between the left and right circular polarizations can be expressed for the $L_{3}$ edge $\left(2 p_{3 / 2}\right)$

as

$$
\begin{aligned}
\Delta I_{L 3} & \propto\left|\left\langle 2,2\left|\mathbf{r}_{+}\right| 3 / 2,1 / 2\right\rangle\right|^{2} h_{+2}+\left|\left\langle 2,1\left|\mathbf{r}_{+}\right| 3 / 2,-1 / 2\right\rangle\right|^{2} h_{+1}-\left(\left|\left\langle 2,0\left|\mathbf{r}_{+}\right| 3 / 2,-3 / 2\right\rangle\right|^{2}-\left|\left\langle 2,0\left|\mathbf{r}_{-}\right| 3 / 2,1 / 2\right\rangle\right|^{2}\right) h_{0} \\
& -\left|\left\langle 2,-1\left|\mathbf{r}_{-}\right| 3 / 2,-1 / 2\right\rangle\right|^{2} h_{-1}-\left|\left\langle 2,-2\left|\mathbf{r}_{-}\right| 3 / 2,-3 / 2\right\rangle\right|^{2} h_{-2} \\
& =6 h_{+2}+6 h_{+1}+2 h_{0}-6 h_{-}-18 h_{-2}
\end{aligned}
$$

(11)

Similarly the difference in the absorption intensity for the transition of $2 \mathrm{p}_{1 / 2}$ to $3 \mathrm{~d}$ orbitals is expressed as

$\Delta I_{L 2} \propto 12 h_{+2}+3 h_{+1}-2 h_{0}-3 h_{-1}$

As a result, the difference in the intensity of the edge peak can be obtained when the helicity of the circularly polarized light or magnetic field is switched. This difference in the spectra measured with two circular polarizations in the magnetic field is referred to as X-ray magnetic circular dichroism (XMCD). When PEEM is applied for the measurement of an XMCD map of $\mathrm{L}_{2,3}$ edge spectra of a surface, then the magnetic domain distribution can be obtained, which has already been reviewed. ${ }^{4,38}$

UV-lasers are also expected to be a promising light source for PEEM, due to their high intensity and pulse structure. However, if an excessively large intensity is emitted, then the 
PEEM image is blurred, due to the space charge and charge-up effects. ${ }^{39,40}$ Instead, a two-photon photoemission (2PPE) process using a short pulse and intense laser with each photon energy less than ionization threshold can be employed. 2PPE provides images that are sensitive to the intermediate electronic states, such as plasmon excitation to which the first photon pulse excites the electron. ${ }^{41}$ By changing the time delay of the second pulse, a time-resolved PEEM image of the dynamic response of the surface, such as relaxation processes of localized surface plasmons, can be obtained. ${ }^{42-45}$ Pump-probe experiments reveal the relaxation time of the intermediate state and the spatial propagation. ${ }^{46}$

Photoelectrons and X-rays can penetrate into the bulk and the escape depth of photoelectrons is a few nanometers. In this sense PEEM and EXPEEM are not strictly surface sensitive. An electron excited by metastable He* through a Penning ionization process gives a microscopic image sensitive to only the topmost electron state. ${ }^{47,48}$ This technique is called metastable electron emission microscopy (MEEM). When a He atom is excited to a triplet state with an electronic structure of (1s 2s), where two electrons have the same spin direction, it forms a metastable atom with a long lifetime. When He* approaches a surface, an electron transfers from the surface to the 1s hole of He*, accompanied by emission of the electron from 2s level, such as an Auger electron process. This is referred to as a Penning ionization process. The kinetic energy is dependent on the energy difference between the $1 \mathrm{~s}$ core hole and the 
valence electron of the surface; therefore, the electronic structure can be obtained from analysis of the emitted electron. The advantage of Penning ionization is that the interaction of He* is limited to the topmost electron state, due to the impenetrability of $\mathrm{He}^{*}$ and MEEM yields a different contrast from PEEM. ${ }^{49}$

\subsection{PEEM and EXPEEM Optics}

Fig. 3(a) and Fig. 4 shows PEEM and EXPEEM apparatuses drawn schematically. EXPEEM contains the EA part. The main difference of PEEM and EXPEEM from TEM is the objective lens. Since the ejected photoelectron is widely dispersed, the function of the objective lens in PEEM is to collect as many ejected electrons as possible. ${ }^{50,51}$ For this purpose, a high voltage $(10-20 \mathrm{kV})$ is applied between the first electrode of the objective lens and the sample, where electrons are accelerated in one direction by the high voltage, as shown in Fig. 9. In order to create an image, the electron is focused by second and third electrodes placed behind.

The spatial resolution of PEEM is determined by the aberration of the objective lens as described by:

$$
\begin{aligned}
& d^{2}=d_{D}{ }^{2}+d_{S}{ }^{2}+d_{c}{ }^{2}, \\
& d_{D}=0.61 \frac{\lambda_{0}}{\alpha_{0}}, d_{S}=\frac{1}{2} C_{s} \alpha_{0}{ }^{3}, d_{c}=C_{c} \frac{\Delta E}{E_{0}} \alpha_{0}
\end{aligned}
$$


where aberration $d$, is a function of the acceptance angle $\alpha_{0}$, and thus, there is an optimal $\alpha_{0}$ for a spatial resolution. A spatial resolution of $10 \mathrm{~nm}$ is usually achieved in a conventional PEEM and one that has aberration corrected can provide a spatial resolution of a few nanometers.

Since the amount of core-shell photoelectrons excited by X-ray is small, a larger $\alpha_{0}$ is required in order to obtain a good EXPEEM signal at the sacrifice of the spatial resolution. Therefore, improvement of the chromatic and spherical aberration coefficients of the object lens is important to obtain high spatial resolution and a good S/N ratio image for EXPEEM.

Several aberration correction methods for the objective lens have been developed, such as the mirror corrector, ${ }^{52-54}$ a mesh or foil objective lens, ${ }^{55,56}$ multipole electric and magnetic fields, ${ }^{57,58}$ and moving focus method. ${ }^{59}$

\subsection{Energy analyzer for PEEM}

\subsubsection{Chemically sensitive PEEM}

The EXPEEM requires X-ray exciting the core electron and the energy analyzer as shown in

Fig. 4b. Fig. 10 shows the kinetic energy distribution of photoelectrons excited by high energy X-rays. The largest electron peak appears at close to $0 \mathrm{eV}$, due to the strong secondary electron emission. The primary photoelectron peak appears at a certain energy determined by

$$
E_{k i n}=h v-E_{B}
$$

where $h v$ is the photon energy and $E_{B}$ is the binding energy of the core electron for an X-ray absorbing atom. The image contrast is not only dependent on the peak height shown by S in Fig. 
10, but also on the background (B), which is mainly composed of secondary electrons. If light atoms are absorbed on the surface of heavy elements, then the region where the light atoms are absent appears brighter, even if the photoelectrons of the light atoms are selected, because more secondary electrons are excited from heavier elements. Fig. 11 shows EXPEEM images of Au islands on a Ta substrate using photoelectrons with kinetic energies in the $\mathrm{O} 1 \mathrm{~s}$ region. Fig. 11a shows a secondary electron X-ray PEEM image with kinetic energy $=0 \mathrm{eV}$ in order to show the sample outline. The bright regions are Au islands, while the remainder is oxidized Ta. When the $\mathrm{O}$ 1s X-ray photoelectron peak is selected, the Au regions are still brighter, as shown in

Fig. 11b. This is not due to the oxygen present on the Au regions, but because more secondary electrons are emitted from the Au regions than $\mathrm{O}$ 1s electrons from the Ta region as mentioned above. When photoelectrons with $5 \mathrm{eV}$ higher kinetic energy than the O1s photoelectron peak are selected, the Ta region becomes much darker, while the Au region retains its brightness as shown in Fig. 11. Consequently it is necessary to measure the background emission to obtain the actual element distribution. A high contrast image is sometimes obtained when a higher kinetic energy peak is selected, because of a lower background as shown in Fig. $10 . \quad$ Fig. 12 shows an EXPEEM image of $\mathrm{Au}$ islands on Ta substrate obtained using different kinetic 
energies. The Au regions are brighter at kinetic energy $=188 \mathrm{eV}$. This is not only because the kinetic energy corresponds to $\mathrm{Au} 3 \mathrm{~d}_{5 / 2}$, but because more background secondary electrons comes from the Au region than from the Ta region. When Ta $3 \mathrm{p}_{3 / 2}$ electron is selected, the Ta substrate region becomes slightly brighter, but the contrast is not so high due to the strong background emission from the Au regions. For the electron selected at kinetic energy $=653 \mathrm{eV}$, which corresponds to Ta $3 \mathrm{~d}_{5 / 2}$ photoelectrons, the Ta substrate becomes much brighter, because the secondary electron emission becomes less than the emission at lower kinetic energy as shown in Fig. 10.

\subsubsection{Energy analyzer for EXPEEM (other than a Wien filter)}

Among several EAs used for EXPEEM, the hemispherical energy analyzer are the most popular as shown in Fig. 4b.. The pass energy of a photoelectron is determined by

$$
E_{0}=\frac{e V_{D}}{\left(\frac{R_{2}}{R_{1}}-\frac{R_{1}}{R_{2}}\right)}
$$

where $R_{1}, R_{2}$ are the inner and outer radii of the analyzer electrodes, respectively. The energy

resolution is expressed as

$$
\Delta E_{A}=\frac{w E_{0}}{2 \mathrm{r}_{0}}+\frac{1}{4} \alpha^{2} E_{0}
$$

where $\alpha$ is the acceptance angle.

In order to remove the chromatic and spherical aberrations in the filter, an asymmetric tandem type hemispherical EA has been developed, referred to as NanoESCA. ${ }^{60,61}$ 
In the SMART instrument built at BESSY II, the German synchrotron facility, ${ }^{54,62}$ an omega type EA has been installed that is suitable for the analysis of high energy electrons. 15 $\mathrm{keV}$ electrons are deflected 4 times by a magnetic field in the omega type EA. The second order aberrations can be cancelled by symmetry and the 6 and 12 pole elements between the sector magnets. ${ }^{54,62}$

The hemispherical and omega type EAs require a bent optical axis, which is a disadvantage for adjustment of the optical axis in microscopy. The following time of flight (TOF) and Wien filter type EAs are collinear type ones that are beneficial in the adjustment of the optical axis and lens conditions in microscopy.

A TOF type EA utilizes the arrival time difference between charged particles traveling through the drift chamber. ${ }^{63}$ The TOF EA requires a pulse light source such as laser, synchrotron radiation or a chopper that causes the simultaneous start of different energy electrons before the TOF drift tube. The transit time $\tau$ is given by $\tau=\frac{L_{d}}{v}=\frac{L_{d}}{\sqrt{2 E_{d} / m}}$ where $L_{d}$ is the TOF tube length and $E_{d}$ is the kinetic energy of the electron in the TOF tube. Temporal dispersion is given as 
$\frac{d \tau}{d E_{d}}=-\frac{L_{d}}{2 \sqrt{2 E_{d}^{3} / m}}$

If a scintillator screen (fastest at a few nanoseconds) is utilized, then the decay time may limit the energy resolution. A delay line detection system improves the time resolution. ${ }^{64}$ Min et al. applied TOF-PEEM with a femtosecond pulse laser to a Ag-coated Si nanostructure and found plasmon-resonance photoemission with a unique time evolution. ${ }^{65}$ Recently sub-femtosecond TOF-PEEM with $25 \mathrm{~nm}$ spatial resolution and $50 \mathrm{meV}$ energy resolution has been reported and the nano-localized plasmon field in the Ag film is investigated. ${ }^{66}$

\subsubsection{Wien filter energy analyzer for EXPEEM}

The other collinear EA available is a Wien filter as shown in Fig. 13, The Wien filter was invented by Wilhelm Wien more than a hundred years ago, ${ }^{67}$ but it is not so often used as an electron energy filter, probably due to the fringe effect. In a Wien filter, the magnetic and electric fields are applied perpendicularly to each other.

The force exerted on an electron traveling through a Wien filter is expressed as:

$\mathbf{F}=e \mathbf{E}+e \mathbf{v} \times \mathbf{B}$

Because the electron kinetic energy can be written as $E=\frac{1}{2} m_{0}|\mathbf{v}|^{2}$, the electron is deflected by the magnetic field according to its velocity. When the Wien condition $\mathbf{F}=e \mathbf{E}+e \mathbf{v} \times \mathbf{B}=0$, is satisfied the electron path is straight. By placing a slit at the exit of the Wien filter, electrons 
with a specific energy can be selected that satisfy the Wien condition. Since it does not contain mass number in equation (18), the Wien filter has been used as both electron and ion energy analyzers. In addition, it can be used as a spin rotator ${ }^{68}$ and a wave shifter. ${ }^{69}$ By adjusting the higher harmonic term of the electric and magnetic fields in the Wien filter, aberrations up to the higher order term can be reduced. ${ }^{70-72}$ The numerical solutions for correction of the second order aberration terms have been discussed. ${ }^{73}$ Here, we discuss the aberration correction of the Wien filter. The Wien conditions are given by the path length $L$, and the initial kinetic energy or path energy $\phi_{0}$ : $E_{1}=\frac{2 \pi \sqrt{2} \phi_{0}}{L}, B_{1}=\frac{2 \pi}{L} \sqrt{\frac{m_{0} \phi_{0}}{e}}$.

Energy resolution is expressed as:

$$
\Delta E=\pi\left(w \phi_{0} / \sqrt{2} L\right)
$$

Thus, a small path energy $\phi_{0}$, and large $L$ provide high energy resolution. The photoelectron is retarded to $50-150 \mathrm{eV}$. In the Wien filter, the inlet and outlet slits should be at the focal planes. The middle of the filter should be the image plane and the maximum height of the orbital $h_{\max }=\frac{L \tan \alpha_{0}}{\pi}$. 
is given at the middle of the Wien filter:

The field of view is limited by $h_{\max }$. Therefore, to obtain high energy resolution and a large field of view, a large $h_{\max }$ is required. In this case the aberration in the Wien filter must be reduced for the electron traveling away from the optical axis.

3.2.4 Principle of aberration correction in the Wien filter ${ }^{74,75}$

The electron orbit can be expressed by a Lagrangian equation:

$\frac{d}{d z}\left(\frac{\partial F}{\partial \vec{r}^{\prime}}\right)-\frac{\partial F}{\partial \vec{r}}=0$

$F$ is the Lagrangian, which is expressed as:

$F\left(u, \bar{u}, u^{\prime}, \bar{u}^{\prime}\right)=\sqrt{\Phi(u, \bar{u})\left(1+\left|u^{\prime}\right|^{2}\right)}-\sqrt{\left.\frac{e}{2 m_{0}} A_{z}(u, \bar{u})\right)}$

$u=x+i y$

$u^{\prime}=x^{\prime}+i y^{\prime}=\frac{\partial x}{\partial z}+i \frac{\partial y}{\partial z}$

$\bar{u}=x-i y$

$\bar{u}^{\prime}=x^{\prime}-i y^{\prime}$

$\Phi(u, \bar{u})=-\sum_{m=0}^{\infty} \phi_{m c} \frac{u^{m}+\bar{u}^{m}}{2}$ 
where $\Phi(u, \bar{u})$ is the real electrostatic potential and

$\phi_{m c}$ is the mth order coefficient for the electric field.

The magnetic field $\boldsymbol{B}$, can be expressed by a magnetic scalar potential $\Psi$, as

$\boldsymbol{B}=\nabla \times \boldsymbol{A}=-\mu_{0} \nabla \Psi$

$\mu_{0} \Psi(u, \bar{u})=-\sum_{m=0}^{\infty} \psi_{m c} \frac{u^{m}-\bar{u}^{m}}{2 i}$

(27)

$\Phi(x, y)=\phi_{0}-\phi_{1 c} x-\phi_{2 c}\left(x^{2}-y^{2}\right)-\phi_{3 c} x\left(x^{2}-3 y^{2}\right)-\phi_{4 c}\left(x^{4}-6 x^{2} y^{2}-y^{4}\right)-\cdots$

$\mu_{0} \Psi(x, y)=-\psi_{1 s} y-2 \psi_{2 s} x y-\psi_{3 s} y\left(3 x^{2}-y^{2}\right)-4 \psi_{4 s}\left(x^{2}-y^{2}\right)-\cdots$

(28)

$A_{z}(u, \bar{u})=-\sum_{m=0}^{\infty} \psi_{m s} \frac{u^{m}+\bar{u}^{m}}{2}$

(29)

$\phi_{0}, \phi_{1 c}, \phi_{2 c}$ and $\psi_{1 s}, \psi_{2 s}, \psi_{3 s}$ are the coefficients of the Fourier expansions for the electric and magnetic fields. Thus, the Lagrangian is also expanded in the polynomial of $u$

$F=F_{0}+F_{1}+F_{2}+F_{3}+F_{4}$

(30) 


$$
\begin{aligned}
F_{1}= & -\frac{1}{4 \phi_{0}{ }^{1 / 2}}\left(\phi_{1 c}-v_{0} \psi_{1 s}\right)(u+\bar{u}), \\
F_{2}= & -\frac{1}{32 \phi^{3 / 2}}\left\{\phi_{1 c}{ }^{2}(u+\bar{u})^{2}-16 \phi_{0}{ }^{2}\left|u^{\prime}\right|^{2}+8 \phi_{0}\left(\phi_{2 c}-v_{0} \psi_{2 s}\right)\left(u^{2}+\bar{u}^{2}\right)\right\} \\
F_{3}= & -\frac{1}{128 \phi_{0}^{5 / 2}}\left\{\phi_{1 c}{ }^{3}(u+\bar{u})^{3}+8 \phi_{0} \phi_{1 c} \phi_{2 c}(u+\bar{u})\left(u^{2}+\bar{u}^{2}\right)-16 \phi_{0}{ }^{2} \phi_{1 v}(u+\bar{u})\left|u^{\prime}\right|^{2}+32 \phi_{0}{ }^{2}\left(\phi_{3 c}-v_{0} \psi_{3 c}\right)\left(u^{3}+\bar{u}^{3}\right)\right\} \\
F_{4}= & -\frac{1}{2048 \phi_{0}{ }^{7 / 2}}\left\{-5 \phi_{1 c}{ }^{4}(u+\bar{u})^{4}+48 \phi_{0} \phi_{1 c}{ }^{2} \phi_{3 c}(u+\bar{u})^{2}\left(u^{2}+\bar{u}^{2}\right)+\right. \\
& 64 \phi_{0}{ }^{2}{\phi_{2 c}}^{2}{ }^{2}\left(u^{2}+\bar{u}^{2}\right)+128 \phi_{0} \phi_{1 c} \phi_{3 c}{ }^{3}(u+\bar{u})\left(u^{3}+\bar{u}^{3}\right)+ \\
& 32 \phi_{0}{ }^{2}{\phi_{1 c}}^{2}(u+\bar{u})^{2}\left|u^{\prime}\right|^{2}+256 \phi_{0}{ }^{3} \phi_{2 c}\left(u^{2}+\bar{u}^{2}\right) u u^{\prime}+ \\
& \left.256 \phi_{0}{ }^{4}\left|u^{\prime}\right|^{4}+512 \phi_{0}{ }^{3}\left(\phi_{4 c}-v_{0} \psi_{4 s}\right)\left(u^{4}+\bar{u}^{4}\right)\right\}
\end{aligned}
$$

In Cartesian coordinate,

$$
\begin{aligned}
F_{1}= & -\frac{1}{4 \phi_{0}{ }^{1 / 2}}\left(\phi_{1 c}-v_{0} \psi_{1 s}\right) 2 x, \\
F_{2}= & -\frac{1}{32 \phi_{0}{ }^{3 / 2}}\left\{4{\phi_{1 c}}^{2} x^{2}-16 \phi_{0}{ }^{2}\left(x^{\prime 2}+y^{\prime 2}\right)\right. \\
& \left.+16 \phi_{0}\left(\phi_{2 c}-v_{0} \psi_{2 s}\right)\left(x^{2}-y^{2}\right)\right\} \\
F_{3}= & -\frac{1}{128 \phi_{0}{ }^{5 / 2}}\left\{8 \phi_{1 c}{ }^{3} x^{3}+32 \phi_{0} \phi_{1 c} \phi_{2 c} x\left(x^{2}-y^{2}\right)\right. \\
& \left.-32 \phi_{0}{ }^{2} \phi_{1 c} x\left(x^{\prime 2}+y^{\prime 2}\right)+64 \phi_{0}{ }^{2}\left(\phi_{3 c}-v_{0} \psi_{3 s}\right)\left(x^{3}-3 x y^{2}\right)\right\} \\
\mathrm{F}_{4}= & -\frac{1}{2048 \phi_{0}{ }^{7 / 2}}\left\{80 \phi_{1 c}{ }^{4} x^{4}+384 \phi_{0} \phi_{1 c}{ }^{2} \phi_{2 c} x^{2}\left(x^{2}-y^{2}\right)\right. \\
& +128 \phi_{0}{ }^{2} \phi_{2 c}{ }^{2}\left(x^{2}-y^{2}\right)+512 \phi_{0} \phi_{1 c} \phi_{3 c}{ }^{3} x\left(x^{3}-3 x y^{2}\right) \\
& \left.+128 \phi_{0}{ }^{2} \phi_{1 c}{ }^{2} x^{2}\left(x^{\prime 2}+y^{\prime 2}\right)+256 \phi_{0}{ }^{3} \phi_{2 c}{ }^{2} x^{2}-y^{2}\right)\left(x^{\prime 2}+y^{\prime 2}\right) \\
& \left.+256 \phi_{0}{ }^{4}\left(x^{\prime 2}+y^{\prime 2}\right)^{2}+1024 \phi_{0}{ }^{3}\left(\phi_{4 c}-v_{0} \psi_{4 s}\right)\left(x^{4}-6 x^{2} y^{2}+y^{4}\right)\right\}
\end{aligned}
$$

where $x$ is the energy dispersion direction.

For simplification, the Fourier components are reduced to the $e_{2}, e_{3}, e_{4}, b_{2}, b_{3}$, and $b_{4}$ terms hereinafter: 


$$
\begin{aligned}
& e_{2}=\frac{\phi_{2 c}}{\phi_{1 c}} R_{0}, e_{3}=\frac{\phi_{3 c}}{\phi_{1 c}} R_{0}{ }^{2}, e_{4}=\frac{\phi_{4 c}}{\phi_{1 c}} R_{0}{ }^{3} \\
& b_{2}=\frac{\psi_{2 s}}{\psi_{1 s}} R_{0}, b_{3}=\frac{\psi_{3 s}}{\psi_{1 s}} R_{0}{ }^{2}, b_{4}=\frac{\psi_{4 s}}{\psi_{1 s}} R_{0}{ }^{3}
\end{aligned}
$$

$$
\text { where } \quad R_{0}=2 \phi_{0} / \phi_{1 c}
$$

and the Wien condition is given as

$$
e_{2}-b_{2}=-\frac{1}{4}
$$

In order to derive the first-order trajectory, the Lagrangian is used up to the second-order power series:

$$
F=F_{0}+F_{1}+F_{2}
$$

$$
u^{\prime \prime}+\frac{1}{\phi_{0}}\left(\frac{\phi_{1 c}^{2}}{8 \phi_{0}}(u+\bar{u})+\left(\phi_{2 c}-v_{0} \psi_{2 s}\right) \bar{u}\right)=-\frac{1}{2 \phi_{0}}\left(\phi_{1 c}-v_{o} \psi_{1 s}\right)
$$

The right hand term is the first derivative of the potential or deflection force for the electron in the dispersion direction ( $x$ direction) on the optical axis. For the electron that satisfies the Wien condition $\left(\phi_{1 c}-v_{o} \psi_{1 s}\right)$, the left-side term becomes zero. Off-axial electrons should meet together again at the focal point (a stigmatic condition). Because $\mathrm{u}=x+i y$, the stigmatic condition is fulfilled when the real and imaginary parts of the equation have the same expression. 


$$
\frac{\phi_{1 c}{ }^{2}}{8 \phi_{0}}=-\left(\phi_{2 c}-v_{0} \psi_{2 s}\right)
$$

(36)

Finally, the equation is equal to

$$
u^{\prime \prime}+\frac{\phi_{1 c}^{2}}{8 \phi_{0}^{2}} u=0
$$

$$
u=\exp \left(i \frac{z}{L} \pi\right)
$$

$$
L=\frac{2 \sqrt{2} \pi \phi_{o}}{\phi_{1 c}}=\frac{2 \sqrt{2} \pi \phi_{o}}{E_{1}}
$$

Consequently, equation(21) is derived.

In order to reduce the aberrations, the higher order term is taken into account.

The aberration coefficients of the Wien filter are derived using the third order Lagrangian under the Wien condition and the stigmatic condition.

$F=F_{0}+F_{1}+F_{2}+F_{3}$

$\Delta u_{2}{ }^{\prime \prime}+\frac{\phi_{1 c}{ }^{2}}{8 \phi_{0}{ }^{2}} \Delta u_{2}=\frac{1}{\phi_{0}^{1 / 2}}\left\{\nabla_{u} F_{3}-\left(\nabla_{u^{\prime}} F_{3}\right)^{\prime}\right\}$ 
where $\Delta u_{2}$ is the second order aberration.

$\Delta u_{2}$ can be expressed at the image plane: ${ }^{75}$

$\Delta u_{2}\left(z_{i}\right)=\frac{1}{\phi_{0}^{1 / 2}} \int_{z_{0}}^{z_{1}}\left(\Delta_{u_{0}^{\prime}} F_{3}^{i n t}\right) d z$

where $z_{0}$ is the entrance position.

$F_{3}{ }^{\text {int }}$ is the third order Lagrangian in the interaction or on the first order trajectory, which is defined as:

$F_{3}{ }^{i n t}\left(u_{0}, \overline{u_{o}} u_{0}{ }^{\prime}, \overline{u_{o}{ }^{\prime}} ; z\right)=F_{3}\left(u_{0} g(z)+u_{0}{ }^{\prime} h(z), \overline{u_{0}} g(z)+\overline{u_{0}{ }^{\prime}} h(z), u_{0} g^{\prime}(z)+u_{0}{ }^{\prime} h^{\prime}(z), \overline{u_{0}} g^{\prime}(z)+\overline{u_{0}} h^{\prime}(z)\right)$

where $g(z)$ and $h(z)$ are two fundamental solutions of (35) with initial conditions of $g\left(z_{0}\right)=1 ; g^{\prime}\left(z_{0}\right)=0 ; h\left(z_{0}\right)=0 ; h^{\prime}\left(z_{0}\right)=1$.

Analytical integration of (42) indicates dependence on the aberration coefficients $C$ (pqrs), as defined by the following equation.

$$
\Delta u_{2}=\sum C(p q r s) u_{0} \bar{p}^{q} u_{0}{ }^{r} \overline{u_{0}{ }^{\prime}} s
$$

where $\Delta u_{2}$ is the n-th order aberration.

The second order aberration coefficients, $C_{2}$ (pqrs)'s, are expressed by the reduced Fourier components of the electric and magnetic fields,

$$
\begin{aligned}
& C_{2}(2000)=-\frac{2}{3 R_{0}} b_{2}, C_{2}(1100)=-\frac{1}{3 R_{0}}\left(3+4 b_{2}\right), C_{2}(0200)=-\frac{2}{R_{0}}\left(b_{2}+2\left(e_{3}-b_{3}\right)\right), \\
& C_{2}(0020)=-\frac{2 R_{0}}{3}\left(3+4 b_{2}\right), C_{2}(0011)=-\frac{4 R_{0}}{3}\left(3+4 b_{2}\right), C_{2}(0002)=-8 R_{0}\left(b_{2}+2\left(e_{3}-b_{3}\right)\right) .
\end{aligned}
$$


Here we assume that the point source, $C_{2}$ (pqrs), can be negligible, unless $p \neq 0, q \neq 0$. Under these conditions and the Wien condition, $C_{2}($ pqrs $)=0 \quad$ when $e_{2}=-1, b_{2}=-3 / 4, e_{3}-b_{3}=3 / 8$. The conditions agree with those reported by Rose. ${ }^{71}$ Thus, we refer to the condition as the Rose condition.

Similarly, the third order aberration correction conditions can be obtained. The condition can be determined by solving the fourth order Lagrangian

$F=F_{0}+F_{1}+F_{2}+F_{3}+F_{4}$

The third order aberration $\Delta u_{3}$, is given by:

$\Delta u_{3}{ }^{\prime \prime}+\frac{\phi_{1 c}{ }^{2}}{8 \phi_{0}{ }^{2}} \Delta u_{3}=\frac{1}{\phi_{0}^{1 / 2}}\left[D_{2}\left(\nabla_{u} F_{3}\right)-\left\{D_{2}\left(\nabla_{u^{\prime}} F_{3}\right)^{\prime}+\nabla_{u} F_{4}-\left(\nabla_{u^{\prime}} F_{4}\right)^{\prime}\right]\right.$

(46)

where

$D_{2}=\Delta u_{2} \frac{\partial}{\partial u}+\Delta \bar{u}_{2} \frac{\partial}{\partial \bar{u}}+\Delta u_{2}^{\prime} \frac{\partial}{\partial u^{\prime}}+\Delta \bar{u}_{2}{ }^{\prime} \frac{\partial}{\partial \bar{u}^{\prime}}$,

Equation (46) is linear, so that it can be divided into two parts that are related to only $F_{3}$ and

$F_{4}$, respectively.

$\Delta u_{3 A}{ }^{\prime \prime}+\frac{\phi_{1 c}{ }^{2}}{8 \phi_{0}{ }^{2}} \Delta u_{3 A}=\frac{1}{\phi_{0}{ }^{1 / 2}}\left[D_{2}\left(\nabla_{u} F_{3}\right)-\left\{D_{2}\left(\nabla_{u^{\prime}} F_{3}\right)\right\}\right]$

$\Delta u_{3 B}{ }^{\prime \prime}+\frac{\phi_{1 c}{ }^{2}}{8 \phi_{0}^{2}} \Delta u_{3 B}=\frac{1}{\phi_{0}^{1 / 2}}\left[\nabla_{u} F_{4}-\left(\nabla_{u^{\prime}} F_{4}\right)^{\prime}\right]$ 
Both parts are independently solved at the image plane $\left(\mathrm{z}=\mathrm{z}_{\mathrm{i}}\right)$.

$$
\begin{aligned}
& \Delta u_{3 A}\left(z_{i}\right)=\frac{1}{{\phi_{0}}^{1 / 2}} \int_{z_{o}}^{z_{i}} D_{2}{ }^{i n t}\left(\nabla_{u_{0}}{ }^{\prime} F_{3}{ }^{i n t}\right) d z \\
& \Delta u_{3 B}\left(z_{i}\right)=\frac{1}{{\phi_{0}}^{1 / 2}} \int_{z_{o}}^{z_{i}}\left(\nabla_{u_{0}} F^{{ }^{i n t}}\right) d z
\end{aligned}
$$

(49)

where the differential operator $D_{2}{ }^{i n t}$, is given by

$$
D_{2}^{i n t}=\Delta u_{2}^{i n t}(z) \frac{\partial}{\partial u_{0}}+\Delta \bar{u}_{2}{ }^{i n t}(z) \frac{\partial}{\partial \bar{u}_{0}}+\Delta u_{2}^{i n t}\left(z^{\prime}\right) \frac{\partial}{\partial u_{0}{ }^{\prime}}+\Delta \bar{u}_{2}{ }^{i n t}\left(z^{\prime}\right) \frac{\partial}{\partial \bar{u}_{0}{ }^{\prime}} .
$$

Finally, integration of equation (50) and the third order aberration coefficients $C_{3}($ pqrs), are performed. It is again assumed that the point source and all $C_{3}$ (pqrs) coefficients are neglected for $p \neq 0, q \neq 0$.

$$
\begin{aligned}
& C_{3}(0030)=\frac{\pi R_{0}}{24 \sqrt{2}}\left(3+4 b_{2}\right)\left(3+40 b_{2}+60\left(e_{3}-b_{3}\right)\right)-\frac{\pi R_{0}}{8 \sqrt{2}}\left(3+20 b_{2}+12 e_{3}\right) \\
& C_{3}(0021)=\frac{\pi R_{0}}{6 \sqrt{2}}\left(21+66 b_{2}+140 b_{2}{ }^{2}+360 b_{2}\left(e_{3}-b_{3}\right)+360\left(e_{3}-b_{3}\right)^{2}\right)-\frac{\pi R_{0}}{2 \sqrt{2}}\left(5+6 b_{2}+6 b_{2}{ }^{2}\right) \\
& C_{3}(0012)=\frac{\pi R_{0}}{8 \sqrt{2}}\left(3+4 b_{2}\right)\left(3+40 b_{2}+60\left(e_{3}-b_{3}\right)\right)-\frac{3 \pi R_{0}}{8 \sqrt{2}}\left(3+20 b_{2}+12 e_{3}\right) \\
& C_{3}(0003)=\frac{\pi R_{0}}{\sqrt{2}}\left(3+10 b_{2}\right)\left(b_{2}+2\left(e_{3}-b_{3}\right)\right)-\frac{3 \pi R_{0}}{\sqrt{2}}\left(b_{2}+b_{2}{ }^{2}+2 e_{3}+4\left(e_{4}-b_{4}\right)\right)
\end{aligned}
$$

By satisfying the Rose condition, it is difficult to optimize the 4 equations in (51). 
Thus, only the aberration in the energy dispersion direction is eliminated, i.e., the $x$ direction

corresponding to the real part $\Delta u_{3}$.

$$
\begin{aligned}
& \Delta x_{3}=\frac{1}{2}\left(\Delta u_{3}+\Delta \bar{u}_{3}\right) \\
& =\left\{C_{3}(0030)+C_{3}(0021)+C_{3}(0012)+C_{3}(0003)\right\} x^{\prime 3}+\left\{-3 C_{3}(0030)+C_{3}(0021)+C_{3}(0012)-3 C_{3}(0003)\right\} x^{\prime} y^{\prime 2}
\end{aligned}
$$

$\Delta x_{3}=0$ when

$C_{3}(0030)+C_{3}(0021)+C_{3}(0012)+C_{3}(0003)=0$

$-3 C_{3}(0030)+C_{3}(0021)+C_{3}(0012)-3 C_{3}(0003)=0$

The above conditions can be satisfied when

$e_{2}=-1, b_{2}=-3 / 4, e_{3}=0.563, b_{3}=0.188, e_{4}-b_{4}=-0.180$

The conditions are summarized in Table 1.

Table 1. Aberration correction conditions.

\begin{tabular}{|l|l|l|l|}
\hline $\begin{array}{l}\text { Reduced Fourier } \\
\text { components }\end{array}$ & $\begin{array}{l}\text { stigmatic conditions } \\
\text { (Condition A) }\end{array}$ & $\begin{array}{l}\text { Rose conditions } \\
\text { (Condition B) }\end{array}$ & $\begin{array}{l}\Delta x_{3}=0 \\
\text { (Condition C) }\end{array}$ \\
\hline$e_{2}$ & -0.250 & -1 & -1 \\
\hline$b_{2}$ & 0 & $-0.75=(-3 / 4)$ & $-0.75=(-3 / 4)$ \\
\hline$e_{3}$ & 0 & $0.375(=3 / 8)$ & 0.563 \\
\hline$b_{3}$ & 0 & 0 & 0.188 \\
\hline$e_{4}$ & 0 & 0 & -0.180 \\
\hline$b_{4}$ & 0 & 0 & 0 \\
\hline & & & \\
\hline
\end{tabular}

Aberration figures were calculated using three conditions, as follows. A charge simulation

method (CSM) was used, which is a method used to calculate any arbitrary point by placing virtual

electric and magnetic charges at the boundary to simulate the field in a short time. ${ }^{76}$ Firstly, we 
performed ray trace using the ideal electric and magnetic fields. The energy dispersion

direction, i.e., the direction of electron deflection, is set in the $\mathrm{x}$ direction. The aberration

figures of conditions A, B and C are shown in Fig. 14a, b, and c, respectively. Comparing the

blur of the energy dispersion direction for each aberration figure gives approximately 27, 12 and

$3 \mathrm{~mm}$ for conditions $\mathrm{A}, \mathrm{B}$ and $\mathrm{C}$, respectively.

It was confirmed that the aberration was effectively reduced under condition $\mathrm{C}$ in the energy

dispersion direction. For practical application, the $4^{\text {th }}$ order electric and magnetic components, $\mathrm{e}_{4}$ and $\mathrm{b}_{4}$ must be controlled using multipole electrodes and magnetic poles. In order to create $4^{\text {th }}$ order electric and magnetic fields, at least 8 electrodes and magnetic poles are required. The fields that satisfy condition $\mathrm{C}$ are calculated using the CSM. ${ }^{74}$ Fig. 15a shows an aberration figure for 8 electrodes and magnetic poles. The blurring of the aberration figure is approximately $30 \mathrm{~mm}$. The reason for such a large aberration is that the Fourier components higher than the fourth order are not controlled, which results in deterioration of the aberration figure. More electrodes and magnetic poles are added and aberration figures are calculated in order to reduce the higher order Fourier components. A sufficiently small dispersion in the x-direction, as shown in Fig. 15c, is obtained when electrodes and magnetic 12 poles are assumed. Further increase of the electrodes and magnetic poles to 18 provides only slight improvement of the aberration, as shown in Fig. 15d. Therefore, 12 electrodes and magnetic 
poles are the best practical choice for the multipole Wien filter. The remaining small blur occurs due to a fringe effect. In the ideal case, there is no distribution of electric and magnetic fields in the z-direction. However, the real fields gradually increase and decrease along the z-axis in the entrance and exit positions of the Wien filter, which is known as the fringe effect. Fig. 16 a and c show the electron trajectory with and without the fringe effect, respectively. The blur appears in the dispersion direction when the fringe field is present as shown in Figs. Fig. $16 \mathrm{~b}$ and d. In order to observe the fringe effect more clearly, two cases are simulated, as shown in

Fig. 17. A Wien filter lies between two convex lenses and the focal points of the convex lenses are placed at the entrance and exit points of the Wien filter, respectively. Firstly, the point source is placed on the front focal point of the entrance convex lens, as shown in

Fig. 17a. The beam enters the Wien filter parallel to the optical axis. In the ideal case, the beam is focused on the center of the filter, while the focal point moves to the exit side in the real case with a fringe field, because of the weaker field at the entrance region. When condition C is satisfied, the beam passes through the ideal position at the exit plane; however, the beam is not focused on the image plane made by the exit convex lens except in the optical axis, which results in a large blur. The other case is that where parallel beams are focused on the entrance point and result in the image at the center of the Wien filter, as shown in 
Fig. 17b. The beams are again focused at the exit position and are shifted in the dispersion direction, which results in a small blur with a pincushion distortion on the image plane. In order to decrease the fringe effect, the beam should pass through the optical axis near the entrance and exit positions on the focal points coincide with entrance point.

Another disadvantage of the Wien filter is hysteresis of the magnetic poles. Since an iron core electromagnet is usually used in the Wien filter, it has a hysteresis, i.e., the magnetic poles do not produce the same intensity magnetic field at the increasing and decreasing processes. Niimi et $a .^{74}$ attempted to use an air core coil, which has no hysteresis; however, the air core coil has a problem in that the boundary conditions of the electric and magnetic fields do not coincide. In the iron core electromagnets, the iron core can be used as an electrode. The air core electromagnets require that the electrodes should be put out side of them. Otherwise, electrode would affect the magnetic field. This inconsistency in the boundary conditions can be overcome by modifying the shape and size of the electrodes. If the electrodes and air core coils are arranged with the same length, as shown in Fig. 18, then the dipole component of the electric and magnetic fields on the optical axis can not be coincided at the fringe region and the undesired deflection forces is applied on the travelling electron. When the magnetic poles are elongated in the optical axis direction, the magnetic fields are enhanced in the fringe region. In addition, the electric field is reduced by using a tapered electrode. Fig. 19 shows the Wien 
filter with a modified electrodes and magnetic poles and the dipole components of the magnetic and electric fields. Fig. 20 shows the trajectory of the electron beam for the Wien filter with the modified electrodes and magnetic poles and the corresponding aberration figure. The blur becomes as small as that for the iron-core Wien filter. We have constructed such type of Wien filter and installed it in the EXPEEM. Fig. 21 shows the EXPEEM images of Au islands on a Ta substrate obtained using the tapered air-core coil Wien filter. The Au island size is $10 \mu \mathrm{m}$. The Au islands appear brighter when photoelectrons with kinetic energy at $0 \mathrm{eV}$ are selected. The $\mathrm{Au}$ regions become brighter when $\mathrm{Au} 4 \mathrm{f}_{7 / 2}$ is selected, while the Ta substrate becomes brighter when the $\mathrm{Ta} 4 \mathrm{f}_{7 / 2}$ photoelectron peak region is selected.

\subsubsection{Advantages and Disadvantages of PEEM and EXPEEM}

The advantages of PEEM are 1) in situ observation of surface phenomena, 2) less severe damage to the sample, 3) surface mapping, depending on the chemical or physical properties, by the selection of appropriate light sources. The disadvantage is the limited spatial resolution (a few tens of nanometers for conventional apparatus). The best resolution reported in 2009 for PEEM was a few nanometers. ${ }^{77}$ In order to increase the resolution, two improvements are necessary. One is to increase the power of the light source; however, when an excessively intense light source is used, space charge and sample damage must be taken into account. The other improvement is aberration correction that allows a larger acceptance angle as mentioned before. $^{52-59}$ 


\section{Chemical application of EXPEEM}

In the final part of this review, the applications of PEEM and EXPEEM to chemistry are described.

\subsection{In situ observation of surface reactions}

PEEM and EXPEEM can be used to monitor surface reactions, as shown in Fig. $1^{3}$ In situ observation during a reaction is important to understand chemical phenomena on a surface. Photoelectron techniques were previously believed to be irrelevant to in situ studies, because the presence of a gas phase scatters the photoelectrons. However, differential pumping and the aperture at the focal position used in a microscope has enabled photoelectron measurements in the presence of a gas phase. Recently, ambient pressure XPS measurements have become possible using differential pumping of the input lens system prior to the analyzer. ${ }^{78}$ The oxidation reactions of $\mathrm{CH}_{4}, \mathrm{CH}_{3} \mathrm{OH}$ and $\mathrm{CO}$ on noble metal surfaces have been investigated and different surface species and surface reconstructions have been observed under ambient pressure conditions. $^{79}$

A pattern of the adsorbates on a surface is created due to the non-linearity of the surface, where the surface structure changes according to the adsorbate concentration. The surface does not only interact with the gas phase, but also with the other parts of the surface through the diffusion of adsorbates and the dynamic change of the surface structure. Non-linear chemical reactions on surfaces are systematically studied with the aid of theoretical considerations. ${ }^{80,8182,83}$ 
Non-linear behavior of surface reactions can be used to control the catalytic reactions. For example, confinement of the reaction field by a $\mathrm{TiO}_{2}$ wall affects ${ }^{84-86}$ the shape and timing of the chemical reaction. Small domains of less than a few micrometers suppressed the temporal oscillations of $\mathrm{CO}$ and $\mathrm{O}_{2}$ covered states, which indicated that the adsorption probabilities are dependent on the mesoscopic size $(100 \mathrm{~nm}-1 \mu \mathrm{m})$ of the active phase. Chemical modification by the inhomogeneous deposition of Au or Pd on a Pt surface and physical perturbation by changing parameters such as the reaction pressure can vary the spatial temporal pattern formations. ${ }^{87-94}$ For example the chemical reaction originating from the modified surface by Au or Pd transforms the surface state of the remaining region. This is because the adsorption properties and adsorbate coverage of a modified site are different from those of a pure surface. Fig. 22 shows PEEM images of a Pt(110) surface with a 0.3 ML Au covered region whose size was about $100 \mu \mathrm{m}$ under CO oxidation conditions. The CO and oxygen pressure ratio satisfies the condition that the pure Pt surface is fully covered with $\mathrm{O}_{2}$. $\mathrm{O}_{2}$ adsorption is strongly suppressed on the $0.3 \mathrm{ML}$ Au-precovered region, ${ }^{88}$ so that the Au-precovered region is fully covered with $\mathrm{CO}$. $\mathrm{CO}$ can migrate from the Au-precovered region into the pure Pt region where $\mathrm{O}_{2}$ is mainly adsorbed. The $\mathrm{CO}$ concentration wave circled in Fig. 22b migrates into the $\mathrm{O}_{2}$-covered pure Pt region. ${ }^{89}$ This PEEM result has indicated that the surface regions with different adsorption and reaction properties can make communication with each other through 
the diffusion and new reaction patterns can be initiated in the heterogenous part of the surface.

PEEM can also provide information regarding subsurface species. ${ }^{95,96}$ Lauterbach et al. ${ }^{95}$ observed the diffusion of oxygen into the bulk at the subsurface region. The formation of subsurface oxygen occurs from the peripheral region of an oxygen domain and prevails over the entire oxygen covered region. They proposed a subsurface oxygen formation mechanism where dynamic structural changes of Pt play an important role. Fig. 23 shows hydrogen adsorption and absorption processes of Au covered Ta monitored by PEEM. ${ }^{32,}$ 97 When the 20 nm thick Au thin layer is deposited onto the Ta surface and heated to $773 \mathrm{~K}$, AuTa alloy islands are formed and detected by the binding energy shift of Au XPS and the appearance of a Ta peak. 32,97 When the surface is exposed to hydrogen, hydrogen adsorption occurs on the Ta surface. The hydrogen draws electrons from the surface and the work function of the surface increases, and as a result, the surface is darkened. After $10 \mathrm{~min}$, the surface becomes brighter, due to the migration of hydrogen into the subsurface region, which reverses the work function. Similar contrast changes occur in the AuTa alloy island accompanied by $\mathrm{H}_{2}$ adsorption and absorption. The mixing of a small amount of Ta (10-20\%) modifies the Au adsorption properties. After 10 min, the contrast inversion at AuTa alloy island occurs similar to that for the Ta region. However, the brightness change of the AuTa alloy island after $\mathrm{H}_{2}$ exposure is greater than that for the intact Ta region. The contrast difference between the AuTa alloy island and the Ta 
substrate is reduced as shown in Fig. 23(a-3). At 40 min, the introduction of $\mathrm{H}_{2}$ is stopped and the chamber is evacuated. Absorbed hydrogen is removed while the adsorbed hydrogen remains on the surface in Fig. 23(a-4). Consequently, the surface returns to the same contrast level as before the absorption. The formation of AuTa alloy enhances of the absorption and adsorption properties of AuTa alloy island, which might be related to the unique catalytic properties of Au nanoparticles on reducible oxides which showed extremely high activities for CO oxidation. ${ }^{98,99}$

$\mathrm{Ni}_{2} \mathrm{P}$ is a high performance hydrodesulfurization catalyst. ${ }^{100-102}$ The $\mathrm{Ni}_{2} \mathrm{P}$ structure has two types of $\mathrm{Ni}$ atoms that are distinguishable from each other by the number of coordinated $\mathrm{P}$ atoms. ${ }^{103}$ One is tetrahedral $\mathrm{Ni}(1)$ with $4 \mathrm{P}$ atoms and the other is square pyramidal $\mathrm{Ni}(2)$ with $5 \mathrm{P}$ atoms. The $\mathrm{Ni}_{2} \mathrm{P}(0001)$ surface has two surfaces, $\mathrm{Ni}_{3} \mathrm{P}$ and $\mathrm{Ni}_{3} \mathrm{P}_{2}$ surfaces, stacked alternatively. The $\mathrm{Ni}_{3} \mathrm{P}$ and $\mathrm{Ni}_{3} \mathrm{P}_{2}$ surfaces contain only $\mathrm{Ni}(1)$ and $\mathrm{Ni}(2)$, respectively, which are distinguished by scanning tunneling microscopy (STM). ${ }^{103,104}$ Density functional theory (DFT) calculations indicated that the work functions of $\mathrm{Ni}_{3} \mathrm{P}_{2}$ and $\mathrm{Ni}_{3} \mathrm{P}$ terminated surfaces are 5.22 and $4.75 \mathrm{eV}$, respectively. ${ }^{105}$ Fig. 24 shows a PEEM image of the $\mathrm{Ni}_{2} \mathrm{P}(0001)$ surface. A UV cut filter was used with a threshold energy of $5 \mathrm{eV}$. Therefore, the $\mathrm{Ni}_{3} \mathrm{P}_{2}$ with a work function of $5.22 \mathrm{eV}$ becomes dark, while $\mathrm{Ni}_{3} \mathrm{P}$ is bright. Two domains were distinguished with domain sizes of almost $500 \mu \mathrm{m}$, as shown in Fig. 24. 
Locatelli et al. reported auto-heterogenization of an Au-covered Pt surface during the $\mathrm{H}_{2}+\mathrm{O}_{2}$ reaction. ${ }^{106}$ The surface alternated between the oxidized and reduced states. The Au concentration was modulated according to the surface oxidation state. On the oxidized surface, the Au concentration was lowered, while the Au concentration was increased on the reduced surface. The results indicate that Au atoms diffuse on the surface. Similar inhomogenization induced by adsorbates during $\mathrm{O}_{2}+\mathrm{H}_{2}$ reaction conditions has been observed for $\mathrm{K}(\mathrm{Cs})-\mathrm{O}$ on $\operatorname{Rh}(100)^{107}$ and $\mathrm{Rh}$ on $\mathrm{Pt}(100){ }^{108} \mathrm{O}$ has stronger interaction with $\mathrm{K}$ on $\mathrm{Rh}$ and with $\mathrm{Rh}$ on $\mathrm{Pt}$ surfaces. The interaction of adsorbates rearranges the substrate composition. Ternary systems, such as the Rh and $\mathrm{K}$ modified Pt surface, have been studied, which show the co-segregation of Rh and K induced by oxygen adsorption. ${ }^{109}$

The film thickness dependency of oxidation rate was observed on a Mg thin film on W(110) by EXPEEM with a Mg2p peak. ${ }^{110}$ The oxidation rate has correlation with the quantum well state at the Fermi level, which enhances the dissociative adsorption of oxygen.

\subsection{PEEM application to soft material surfaces}

PEEM and EXPEEM have been applied to soft materials such as polymers and biological cells. Soft materials are mainly composed of carbon atoms; therefore, the identification of the carbon species is necessary. Carbon atoms have characteristic 1s X-ray absorption spectral features near the edge which is known as near edge $\mathrm{x}$-ray absorption fine structure 
(NEXAFS). ${ }^{111}$ A block copolymer is a polymer comprising two or more polymer units linked by covalent bonds that have self-organized structures, often called microphase separation structures. Block copolymers often exhibit periodic structures a few nm size such as spherical cylinder, gyroid and lamella structures. The microphase separation structure depends on the composition and temperature. Fig. 25 shows NEXAFS spectra of the poly(styrene) (PS) and poly(methyl methacrylate) (PMMA) copolymer systems. ${ }^{112}$ The PS has a strong peak at 280 $\mathrm{eV}$, assigned to $1 \mathrm{~s}-\pi^{*}$ transition, while PMMA has a slightly stronger peak at $285 \mathrm{eV}$. Thus, when the excitation energy is at $280 \mathrm{eV}$, the PS region is brighter. In the PMMA-rich region, a small PS contribution is always observed. A combination of AFM studies indicates that the nanometer sized PS domains are distributed in PMMA, which can not be resolved by PEEM. ${ }^{112}$ For soft materials, sample damage is not negligible. Wang et al. reported the critical doses for damage to PMMA, fibrinogen (Fg) and PS as 80, 280 and 1230 MGy for $300 \mathrm{eV}$ irradiation at room temperature, respectively. ${ }^{113}$ Yasufuku et al. observed choloroaluminum phthalocyanine (ClAlPc) on $\mathrm{MoS}_{2}$ using PEEM and MEEM. ${ }^{114115}$ They found that diffusion of ClAlPc over the $\mathrm{MoS}_{2}$ surface occurred even at room temperature.

Organic polymers can be used as a scaffold molecule or a vehicle for drug delivery. Such polymers are referred to as biomaterials and they must have an affinity to protein, and thus, adsorptive properties for protein are important. Leung et al. studied protein adsorption on a 
PS-polyactide blend polymer using PEEM and found that the protein selectively adsorbs on the boundary of PS-polyactide. ${ }^{116}$ Similar condensation of blood protein at a polymer boundary is observed. ${ }^{117}$ The interface is the area of the lowest free energy, and therefore, the preferred site for adsorption.

\section{Summary and future prospects}

In this paper PEEM and EXPEEM were reviewed. The advantages of PEEM are in situ observation of surface phenomena, less severe damage to the sample, and surface mapping, depending on the chemical or physical properties, by the selection of appropriate light sources. The disadvantage is limited to the spatial resolution, which is a few tens of nanometers for conventional apparatus. Aberration corrections of the objective lens are important for the improvement of the spatial resolution and the signal intensity which is limited by the acceptance angle, $\alpha$, to reduce the aberration effects. In addition to a mirror corrector and mechanical focusing methods, multipole aberration correctors remain to be improved. Tsuno et al. proposed the application of a multipole Wien filter for the reduction of objective lens aberrations. ${ }^{58}$ Improvement of energy selection is also important for surface chemistry applications. Collinear energy filters, such as a TOF analyzer and a Wien filter analyzer, are promising for ease of alignment of the microscope conditions. The Wien filter has an advantage in the removal of aberrations by controlling the higher order electric and magnetic 
components. Following such improvements, PEEM and EXPEEM are promising tools to reveal chemical reaction mechanisms on surfaces and nanoscale surface dynamics of soft materials. 
${ }^{1}$ H. H. Rotermund, W. Engel, M. Kordesch and G. Ertl (1990). "Imaging of spatio-temporal pattern evolution during carbon monoxide oxidation on platinum." Nature 343(6256): 355.

${ }^{2}$ Y. Yamaguchi, S. Takakusagi, Y. Sakai, M. Kato, K. Asakura and Y. Iwasawa (1999). "X-ray Photoemission Electron Microscopy (XPEEM) as a New Promising Tool for the Real-time Chemical Imaging of Active Surfaces." J.Mol.Catal. 141(1-3): 129-137

${ }^{3}$ H. H. Rotermund (1997). "Imaging of dynamic processes on surfaces by light." Surf.Sci.Report 29: 265.

${ }^{4}$ J. Stohr, Y. Wu, Hermsmeier;B.D, S. M.G., HarpG.R., S. Koranda, Dunham.D and B. P. Tonner (1993). "Element-Specific Magnetic Microscopy with Circularly Polarized X-Rays." Science 259: 658.

${ }^{5}$ F. Guo, H. Sun, T. Okuda, K. Kobayashi and T. Kinoshita (2007). "Surface antiferromagnetic domain structures of $\mathrm{NiO}(001)$ studied using UV photoemission electron microscope." Surface Science 601: 4686-4689.

${ }^{6}$ C. Coluzza and R. Moberg (1995). "Spectromicroscopy and chemical imaging." Surface Review \& Letters 2: 619-641.

${ }^{7}$ H. Yasufuku, Y. Ohminami, T. Tsutsumi, H. Niimi, N. Matsudaira, K. Asakura, M. Kato, Y. Sakai, Y. Kitajima and Y. Iwasawa (2004). "Observation of element specific energy filtered X-ray Photoemission electron microscopy(EXPEEM) images of Au on Ta using a Wien filter type energy analyzer." Jpn.J.Appl.Phys. 43: 7682-7688.

${ }^{8}$ G. Margaritondo (1998). "Photoemission microscopy and its applicaitons to semiconductor science." Jpn.J.Appl.Phys 38 Supple38-1: 8.

9 S. Gunther, M. Marsi, A. Kolmakov, M. Kiskinova, M. Noeske, E. Taglauer, U. A. Schubert, G. Mestl and H. Knozinger (1997). "Photoelectron spectromicroscopymicroscopic study of the spreading behavior of $\mathrm{MoO} 3$ on $\mathrm{TiO} 2$ and Al2O3 Model Supports." J.Phys.Chem. 101: 10004.

${ }^{10}$ H. Ade, A. P. Smith, H. Zhang, G. R. Zhuang, J. Kirz, E. Rightor and A. Hitchcock (1997). "X-Ray Spectromicroscopy of Polymers and Tribological Surfaces At Beamline X1a At 
the Nsls." J. Electron Spectro. Relat. Phenom. 84(1-3): 53-71.

${ }^{11}$ M. Kiskinova and G. Paolucci (1997). "Spectromicroscopy of Complex Interfaces and Real Time Reaction Studies At Elettra." Surf. Sci. 735-743. 377.

12 E. Bauer, C. Koziol, G. Lilienkamp and T. Schmidt (1997). "Spectromicroscopy in a Low Energy Electron Microscope." J. Electron Spectro. Relat. Phenom. 84(1-3): 201-209.

${ }^{13}$ B. P. Tonner, D. Dunham, T. Droubay, J. Kikuma, J. Denlinger, E. Rotenberg and A. Warwick (1995). "The Development of Electron Spectromicroscopy." J. Electron Spectro. Relat. Phenom. 75: 309-332.

14 Griffith and W. Engel (1991). "Historical perspective and current trends in emission microscopy, mirror electron microsocpy and low-energy electron microsopy An introduction to the proceedings of the second international symposium and workshop on emission microscopy and related techqnieus." Ultramicroscopy 36: 1.

${ }^{15}$ E. Ruska and M. Knoll (1931). "Die Magnetische Sammelpule fur schelle electrostahlen." Z. Techn. Physik 12: 389.

${ }^{16}$ E. Bruche (1933). "Electronenmikroskopische Abbildung mit lichtelkrischen electronen." Z.Phys. 86: 448.

17 J. Pohl(1934), " Elektronenoptische Abbildungen mit lichtelektrisch ausgeloesten Elektronen" Z. Tech. Phys. 15 579-581.

${ }^{18}$ G. Somorjai(1994), "Introduction of Surface Chemistry and Catalysis”, Wiley, Wiley Blackwell,

${ }^{19}$ W. Telieps and E. Bauer (1985). "An analytical reflection and emission UHV surface electron microscope." Ultramicroscopy 17: 57.

20 T. Ichinokawa, (1982) " Photoemission electron microscopy” J. Cryst.. Soc. Jpn. 24, 385-389..

${ }^{21}$ S. Jakubith, H. H. Rotermund, W. Engel, A. von Oertzen and G.Ertl (1990). "Spatiotemporal Concentration patterns in a surface reaction: Propagating and Standing Waves, Rotating Spirals and Turbulence." Phys.Rev.Lett. 65: 3013-3015.

${ }^{22}$ W. Engel, M. E. Kordesch, H. H. Rotermund, S. Kubala and A. von Oertzen (1991). "A UHV-compatible photoelectron emission microscope for applications in surface science." Ultramicroscopy 36: 148.

${ }^{23}$ J. Stohr, Y. Wu, Hermsmeier;B.D, S. M.G., HarpG.R., S. Koranda, Dunham.D and B. 
P. Tonner (1993). "Element-Specific Magnetic Microscopy with Circularly Polarized X-Rays." Science 259: 658.

${ }^{24}$ J. Vogel, W. Kuch, M. Bonfim, J. Camarero, Y. Pennec, F. Offi, K. Fukumoto, J. Kirschner, A. Fontaine and S. Pizzini (2003). "Time-resolved magnetic domain imaging by x-ray photoemission electron microscopy." Appl. Phys. Lett., 82: 2299-2301.

${ }^{25}$ G. Beamson, H. Q. Porter and D. W. Turner (1980). "The collimating and magnifying properties of a superconducting field photoelectron spectrometer." J.Phys.E 13: 64-66.

${ }^{26}$ G. Beamson, H. Q. Porter and D. W. Turner (1981). "Photoelectron spectromiscroscopy." Nature 290: 556-561.

${ }^{27}$ D. W. Turner, I. R. Plummer and H. Q. Porter (1984). "Photoelectron Emission Images and Spectra." J. Micro. 136: 259-277.

${ }^{28}$ D. W. Turner, I. R. Plummer and H. Q. Porter (1986). "New Imaging Bandpass Electron-Energy Analyzer." Rev. Sci. Instrum. 57(8): 1494-1500.

${ }^{29}$ B. P. Tonner and G. R. Harp (1989). "Photoyield spectromicroscopy of silicon surfaces using monochromatic synchrotoron radiation." J.Vac.Sci Tehcnol A 7: $1-4$.

${ }^{30}$ B. P. Tonner (1990). "Energy-filtered imaging with electrosctatic optics for photoelectron microscopy." Nucl. Instrum.Methods A291: 60-66.

${ }^{31}$ D.R.Lide ed. (2008) CRC Handbook of Physics and Chemistry, $89^{\text {th }}$ ed.

${ }^{32}$ T. Tsutsumi, T. Miyamoto, H. Niimi, Y. Kitajima, Y. Sakai, M. Kato, T. Naito and K. Asakura (2007). "EXPEEM and its applications to surface and organic materials." Solid State Electron., 51(10): 1360-1366.

${ }^{33}$ T. Nakagawa and T. Yokoyama (2006). "Magnetic Circular Dichroism near the Fermi Level." Phys. Rev. Lett. 96: 237402.

34 T. Nakagawa and T. Yokoyama (2007). "Measurements of threshold photoemission magnetic dichroism using ultraviolet lasers and a phtoelastic modulator." Rev. Sci. Instrum. 78: 023907. 
${ }^{35}$ E. Koch ed.(1983) "Handbook on synchrotron radiation,” North Holand, Amsterdam.

${ }^{36}$ F. de Groot, A. Kotani,(2008), “ Core Level Spectroscopy of Solids,” Boca Raton, CRC Press..

37 A.R.Edmonds(1996) “Angular Momentum in Quantum Mechanics,” Princeton University Press.

${ }^{38}$ C. M. Schneider and G. Schonhense (2002). "Investigating surface magnetism by means of photoexcitation electron emission microscopy " Rep. Prog. Phys. 65(12): R1785-R1839.

${ }^{39}$ G. A. Massey, M. D. Jones and B. P. Plummer (1981). "Space-Charge Aberrations in the Photoelectron Microscope." J.Appl.Phys. 52(6): 3780-3786.

${ }^{40}$ T. Nakagawa, K. Watanabe, Y. Matsumoto and T. Yokoyama (2009). "Magnetic circular dichroism photoemission electron microscopy using laser and threshold photoemission." J.Phys.,C 21:314010

${ }^{41}$ M. Bauer and M. Aeschlimann (2002). "Dynamics of excited electrons in metals, thin films and nanostructures." J. Electron Spectro. Relat. Phenom. 124: 225-243.

${ }^{42}$ D. Bayer, C. Wiemann, O. Gaier, M. Bauer and M. Aeschlimann (2008). "Time-Resolved 2PPE and Time-Resolved PEEM as a Probe of LSP's in Silver Nanoparticles." J. Nanomat.2008; 249514

43 A. Gloskovskii, D. Valdaitsev, S. A. Nepijko, G. Schonhense and B. Rethfeld (2007). "Coexisting electron emission mechanisms in small metal particles observed in fs-laser excited PEEM." Surf.Sci. 601: 4706-4713.

${ }^{44}$ C. Wiemann, D. Bayer, M. Rohmer, M. Aeschlimann and M. Bauer (2007). "Local 2PPE-yield enhancement in a defined periodic silver nanodisk array." Surf.Sci. 601(20): 4714-4721..

${ }^{45}$ F. J. Meyer zu Heringdorf, L. I. Chelaru, S. Mollenbeck, D. Thien and M. Horn-von Hoegen (2007). "Femtosecond photoemission microscopy." Surf.Sci. 601: 4700-4705.

${ }^{46}$ M. Bauer, C. Wiemann, J. Lange, D. Bayer, M. Rohmer and M. Aeschlimann (2007). "Phase propagation of localized surface plasmons probed by time-resolved photoemission electron microscopy." Appl. Phys. A 88: 473-480.

${ }^{47}$ Y. Harada, S. Yamamoto, M. Aoki, S. Masuda, T. Ichinokawa, M. Kato and Y. Sakai (1994). "Surface Spectroscopy with higy spatial resolution using metastable atoms." Nature 372: 657-659. 
${ }^{48}$ Y. Sakai, M. Kato, S. Masuda, Y. Harada and T. Ichinokawa (1998). "Development of a low energy electron microscope with an energy analyzer." Surface Review \& Letters 5(6): 1199-1211.

${ }^{49}$ H. Yasufuku, T. Ibe, M. Okumura, S. Kera, K. K. Okudaira, Y. Harada and N. Ueno (2001). "Diffusion of chloroaluminum phthalocyanine on MoS2 surface detected by photoemission electron microscopy and metastable electron emission microscopy." Journal of Applied Physics 90(1): 213-216.

${ }^{50}$ G. F. Rempfer, W. P. Skoczylas and O. H. Griffith (1991). "Design and Performance of a High-Resolution Photoelectron Microscope." Ultramicroscopy 36(1-3): 196-221.

${ }^{51}$ H. H. Rotermund (1997). "Imaging of dynamic processes on surfaces by light." Surf.Sci.Report 29: 265-364.

52 E.G.Ramberg, (1949) , “Aberration Correction with Electron Mirrors” J.Appl.Phys. 20:183-186

${ }^{53}$ H. Rose and D. Preikszas (1992). "Outline of a Versatile Corrected Leem." Optik 92: 31-44.

${ }^{54}$ R. Wichtendahl, R. Fink, H. Kuhlenbeck, D. Preikszas, H. Rose, R. Spehr, P. Hartel, W. Engel, R. Schlogl, H. J. Freund, A. M. Bradshaw, G. Lilienkamp, T. Schmidt, E. Bauer, G. Benner and E. Umbach (1998). "SMART: An aberration-corrected XPEEM/LEEM with energy filter." Surface Review \& Letters 5: 1249-1256.

${ }^{55}$ H. Matsuda, H. Daimon, M. Kato and M. Kudo (2005). "Approach for simultaneous measurement of two-dimensional angular distribution of charged particles: Spherical aberration correction using an ellipsoidal mesh." Phys. Rev. E 71: 066503.

${ }^{56}$ H. Matsuda, H. Daimon, M. Kato, M. Kudo,(2004) "Spherical aberration correction electrostatic lens, input lens, electron spectroscopic device, photoelectron microsope and measurement system" Japanese Patent 2004-208926.

${ }^{57}$ H. Rose, Optik 85 (1990) 19.

${ }^{58}$ K. Tsuno, D. Ioanoviciu and G. Martinez (2005). "Third order aberration theory of Wien Filters for monochromators and aberration correctors." J.Microsco. 217 (2005) 205-215.

59 T. Koshikawa, H. Shimizu, R. Amakawa, Ikuta, T., T. Yasue and E. Bauer (2005). "A new aberration correction method for photoemission electron microscopy by means of moving forcus." J.Phys.Condens Matter 17: S1371-1380. 
${ }^{60}$ M. Escher, N. Weber, M. Merkel, B. Kromker, D. Funnemann, S. Schmidt, F. Reinert, F. Forster, S. Hufner, P. Bernhard, D. Ziethen, H. J. Elmers and G. Schonhense (2005). "NanoESCA: Imaging UPS and XPS with high energy resolution." J. Electron Spectro. Relat. Phenom. 144: 1179-1182.

${ }^{61}$ M. Escher, N. Weber, M. Merkel, C. Ziethen, P. Bernhard, G. Schonhense, S. Schmidt, F. Forster, F. Reinert, B. Kromker and D. Funnemann (2005). "Nanoelectron spectroscopy for chemical analysis: a novel energy filter for imaging x-ray photoemission spectroscopy." J. Phys.: Cond. Matt., 17: S1329-S1338.

${ }^{62}$ T. Schmidt, U. Groh, R. Fink, E. Umbach, O. Schaff, W. Engel, B. Richter, H. Kuhlenbeck, R. Schlogl, H. J. Freund, A. M. Bradshaw, D. Preikszas, P. Hartel, R. Spehr, H. Rose, G. Lilienkamp, E. Bauer and G. Benner (2002). "XPEEM with energy-filtering: advantages and first results from the smart project." Surf Rev Lett 9(1): 223-232.

${ }^{63}$ H. Spiecker, O. Schmidt, C. Ziethen, D. Menke, U. Kleineberg, R. C. Ahuja, M. Merkel, U. Heinzmann and G. Schoenhense (1998). "Time-of flight photoelectron emission microscopy TOF-PEEM:first results." Nucl. Instrum. Methods 406: 499-506.

${ }^{64}$ A. Oelsner, O. Schmidt, M. Schicketanz, M. Klais, G. Schoenhense, V. Mergel, O. Jagutzki and H. Schmidt-Boecking (2001). "Microscopectrocopy and imaging using a delay line detector in time of flight photoemission microscopy." Rev.Sci.Instrum 72: 3968-3974.

${ }^{65}$ C. K. Min, J. W. Kim and Y. Park (2007). "Femtosecond spectroscopic imaging by time-of-flight photoemission electron microscopy." Surface Science 601: 4722-4726.

${ }^{66}$ J. Lin, N. Weber, A. Wirth, S. H. Chew, M. Escher, M. Merkel, M. F. Kling, M. I. Stockman, F. Krausz and U. Kleineberg (2009). "Time of flight-photoemission electron microscope for ultrahigh spatiotemporal probing of nanoplasmonic optical fields." J. Phys.: Cond. Matt., 21: 314005.

${ }^{67}$ K. Wien (1999). "100 years of ion beams Willy Wien's canal rays." Brazilian J. Phys. 29.

68 T. Kohashi, H. Matsuyama and K. Koike (1995). "A spin rotator for detecting all three magnetization vector components by spin-polarized scanning electron microscopy." $\underline{\mathrm{Rev}}$. Sci Instrum, 66, 5537-5543. 
${ }^{69}$ M. Nicklaus and F. Hasselbach (1993). "Wien filter: A wave-packet-shifting device for restoring longitudinal coherence in charged-matter-wave interferometers." Phys.Rev.A 48(1993)152-160

70 T. T. Tang (1986). "Aberration analysis of a crossed field analyzer." Optik, 74: 51-56.

${ }^{71} \mathrm{H}$. Rose (1987). "The retarding Wien filter as a high performance imaging filter." Optik 77: 26-34.

${ }^{72}$ K. Tsuno (1991). "Aberration analysis of a Wien filter for eletrons." Optik 89: 31-40.

${ }^{73}$ K. Tsuno and J. Rouse (1996). "Simulation of electron trajectories of Wien Filter for HIgh resolution EELS installed in TEM." J.Electron Microsco 45: 417-427.

${ }^{74}$ H. Niimi, W. J. Chun, S. Suzuki, K. Asakura and M. Kato (2007). "Aberration-corrected multipole wien filter for EXPEEM." Rev.Sci.Instrum 78: 063710 1-8.

${ }^{75}$ M. Kato (1997). ” Theory and Design of energy Analyzing Systems for Electron Spectroscopy.” Doctor Thesis of Engineering. The University of Tokyo.

${ }^{76}$ P.E. Hawkes and E. Kasper(1989).” Principles of Electron Optics”, Academic press New York

${ }^{77}$ H. Marchetto, M. Springer, T. Schmidt, H.J. Freund, (2009) "SMART-II: the Next Generation of Aberration Corrected Spectro-Microscopy ", Poster abstracts of FHI MPG 15th Meeting of the Fachbeirat, CP21 Berlin,.

${ }^{78}$ M. Salmeron and R. Schlogl (2008). "Ambient pressure photoelectron spectroscopy: A new tool for surface science and nanotechnology." Surf. Sci. Rep. 63 169-199.

${ }^{79}$ G. Rupprechter and C. Weilach (2008). "Spectroscopic studies of surface-gas interactions and catalyst restructuring at ambient pressure: mind the gap!" J.Phys.-Cond. Matt. 20: 184020.

${ }^{80}$ A. S. Mikhailov and V. S. Zykov (1991). "Kinematical theory of spiral waves in excitable media:Comparizon with numerical simulations." Phys.D. 52: 379-397.

${ }^{81}$ P. Moller, K. Wetzl, M. Eiswirth and G. Ertl (1986). "Kinetic Oscillations in the Catalytic Co Oxidation on Pt(100) - Computer-Simulations." J.Chem.Phys. 85(9): 5328-5336.

82 M. Baer, M. Falcke and M. Eiswirth (1992). "Dispersion relation and spiral rotation in an excitable surface reaction." Physica A 188: 78-88. 
${ }^{83}$ M. Falcke, M. Baer, Engel.H. and M. Eiswirth (1992). "Traveling waves in the CO oxidation on Pt(110): Theory." J.Chem.Phys. 97: 4555-4563.

${ }^{84}$ M. D. Graham, I. G. Kevrekidis, K. Asakura, J. Lauterbach, K. Krischer, H. H. Rotermund and G. Ertl (1994). "Effects of Boundaries on Pattern-Formation - Catalytic-Oxidation of Co on Platinum." Science 264(5155): 80-82.

${ }^{85}$ M. Bar, A. K. Bangia, I. G. Kevrekidis, G. Haas, H. H. Rotermund and G. Ertl (1996). "Composite catalyst surfaces: Effect of inert and active heterogeneities on pattern formation." Journal of Physical Chemistry 100(49): 19106-19117.

${ }^{86}$ M. Kim, M. Bertram, M. Pollmann, A. von Oertzen, A. S. Mikhailov, H. H. Rotermund and G. Ertl (2001). "Controlling chemical turbulence by global delayed feedback: Pattern formation in catalytic CO oxidation on Pt(110)." Science 292(5520): 1357-1360.

${ }^{87}$ M. D. Graham, M. Bar, I. G. Kevrekidis, K. Asakura, J. Lauterbach, H. H. Rotermund and G. Ertl (1995). "Catalysis on Microstructured Surfaces - Pattern-Formation during Co Oxidation in Complex Pt Domains." Phys. Rev. E 52: 76-93.

${ }^{88}$ K. Asakura, J. Lauterbach, H. H. Rotermund and G. Ertl (1995). "Spatiotemporal Concentration Patterns Associated with the Catalytic-Oxidation of Co and Au Covered Pt(110) Surfaces." J. Chem. Phys. 102: 8175-8184.

${ }^{89}$ K. Asakura, J. Lauterbach, H. H. Rotermund and G. Ertl (1994). "Modification of Spatiotemporal Pattern-Formation in an Excitable Medium by Continuous Variation of Its Intrinsic Parameters - Co Oxidation on Pt(110)." Phys. Rev. B 50(11): 8043-8046.

90 J. Lauterbach, K. Asakura and H. H. Rotermund (1994). "Subsurface Oxygen on Pt(100):Kinetics of the Transition from Chemisorbed to Subsurface State and its Reaction with $\mathrm{CO}, \mathrm{H}_{2}$, and $\mathrm{O}_{2}$." Surf.Sci. 313: 52-63..

${ }^{91}$ V. Gorodetskii, J. Lauterbach, H. H. Rotermund, J. H. Block and G. Ertl (1994). "Coupling between adjacent crystal planes in heterogeneous catalysis by propagating reaction-diffusion waves." Nature 370: 276-279.

${ }^{92}$ W. X. Huang, X. H. Bao, H. H. Rotermund and G. Ertl (2002). "CO adsorption on the O-saturated Ag/Pt(110) composite surface: Direct observation of the diffusion of adsorbed CO from strongly bound Pt sites to weakly bound Ag sites." J. Phys. Chem. B 106: 5645-5647. 
${ }^{93}$ J. Lauterbach, K. Asakura, P. B. Rasmussen, H. H. Rotermund, M. Baer, M. D. Graham, I. G. Kevrekidis and G. Ertl (1998). "Catalysis on Mesoscopic Composite Surfaces: Influence of Pd Boundaries on Pattern Formation during CO Oxidation on Pt(110)." Physica D 123: 493-501.

${ }^{94}$ K. Asakura, J. Lauterbach, H. H. Rotermund and G. Ertl (1997). "Spatio-temporal Pattern Formation on the Pt(100) Surface Modified with Submonolayer Gold Coverages." Surf.Sci. 374: 125-141.

95 J. Lauterbach, K. Asakura and H. H. Rotermund (1994). "Subsurface Oxygen on Pt(100):Kinetics of the Transition from Chemisorbed to Subsurface State and its Reaction with CO, $\mathrm{H}_{2}$, and $\mathrm{O}_{2}$." Surf.Sci. 313: 52-63.

${ }^{96}$ N. McMillan, T. Lele, C. Snively and J. Lauterbach (2005). "Subsurface oxygen formation on Pt(100): Experiments and modeling." Catal. Tod. 105: 244-253.

97 K.Asakura, (2010), “Atomic Aspects on Surface Chemical Reaction “, Catal. Tod. in press.

98 M. Haruta (2004). "Gold as anovel catalyst in the 21st century: preparation, working mechanism and applications." Gold Bulletin 37: 27-36..

99 M. S. Chen and D. W. Goodman (2004). "The structure of catalytically active gold on titania." Science 306(5694): 252-255..

100 S. T. Oyama (2003). "Novel Catalysts for Advanced Hydroprocessing transitiom metal Phosphides." J.Catal. 216: 343-352.

101 T. Kawai, S. Sato, W. J. Chun, K. Asakura, K. K. Bando, T. Matsui, Y. Yoshimura, T. Kubota, Y. Okamoto, Y. K. Lee and S. T. Oyama (2003). "In situ XAFS studies on the structure of nickel phosphide catalyst supported on K-USY." Chem.Lett. 32: 956-957.

102 T. Kawai, K. K. Bando, Y. K. Lee, S. T. Oyama, W. J. Chun and K. Asakura (2006). "EXAFS measurements of a working catalyst in the liquid phase: An in situ study of a Ni2P hydrodesulfurization catalyst." J.Cat. 241: 20-24.

${ }^{103}$ M. G. Moula, S. Suzuki, W. J. Chun, S. Otani, S. T. Oyama and K. Asakura (2006). "The first atomic scale observation of a Ni2P(0001) single crystal surface." Chem.Lett 35: 90-91. 
104 S. Suzuki, G. M. Moula, T. Miyamoto, Y. Nakagawa, K. Kinosthita, K. Asakura, S. T. Oyama and S. Otani (2009). "Scanning Tunneling Microscopy and Photoemission Electron Microscopy Studies on Single Crystal Ni2P Surfaces." J. Nanosci. Nanotech. 9(1): 195-201.

105 Q. Li and X. Hu (2006). "First principles study of $\mathrm{Ni}_{2} \mathrm{P}(0001)$ surfaces." Phys.Rev.B 74: 035414.

106 A. Locatelli, C. Sbraccia, S. Heun, S. Baroni and M. Kiskinova (2005). "Energetically driven reorganization of a modified catalytic surface under reaction conditions." J. Amer. Chem. Soc. 127: 2351-2357; A. Locatelli, S. Heun and M. Kiskinova (2004). "Direct observation of reaction induced lateral redistribution of sub-monolayers of Au deposited on a Rh(110) surface." Surf. Sci. 566: 1130-1136.

107 S. Gunther, H. Marbach, R. Imbihl, A. Baraldi, S. Lizzi and M. Kiskinova (2003). "Core level spectroscopy and reactivity of coadsorbed $\mathrm{K}+\mathrm{O}$ layers on reconstructed $\mathrm{Rh}(110)$ surfaces." J.Chem.Phys. 119: 12503-12509.

${ }^{108}$ E. Schutz, F. Esch, S. Gunther, A. M. Schaak, M., M. Kiskinova and R. Imbihl (1999). "Bimetallic catalysts as dissipative sturctures: stationary concentration patterns in the $\mathrm{O}_{2}+\mathrm{H}_{2}$ reaction on a composite $\mathrm{Rh}(110) / \mathrm{Pt}$ surface." Catal.Lett. 63: 13-19.

${ }^{109}$ S. Gunther, H. Marbach, R. Hoyer, R. Imbihl, L. Gregoratti, A. Barinov and M. Kiskinova (2002). "On the origin of stationary concentration patterns in the $\mathrm{H}_{2}+\mathrm{O}_{2}$ reaction on a microstructured $\mathrm{Rh}(110) / \mathrm{Pt}$ surface with potassium." J. Chem. Phys. 117: 2923-2933.

${ }^{110}$ L. Aballe, A. Barinov, A. Locatelli, S. Heun and M. Kiskinova (2004). "Tuning surface reactivity via electron quantum confinement." Phys. Rev. Lett. 93(19): 196103.

111 A. P. Hitchcock, C. Morin, T. Tyliszczak, I. N. Koprinarov, H. Ikeura-Sekiguchi, J. R. Lawrence and G. G. Leppard (2002). "Soft X-ray microscopy of soft matter - Hard information from two softs." Surf. Rev. and Lett, 9(1): 193-201.

112 C. Morin, H. Ikeura-Sekiguchi, T. Tyliszczak, R. Cornelius, J. L. Brash, A. P. Hitchcock, A. Scholl, F. Nolting, G. Appel, D. A. Winesett, K. Kaznacheyev and H. Ade (2001). "X-ray spectromicroscopy of immiscible polymer blends: polystyrene-poly(methyl 
methacrylate)." J. Electro Spec. 121: 203-224.

113 J. Wang, C. Morin, L. Li, A. P. Hitchcock, A. Scholl and A. Doran (2009). "Radiation damage in soft X-ray microscopy." J. Electron Spectro. Relat. Phenom., 170: 25-36.

${ }^{114}$ H. Yasufuku, T. Ibe, M. Okumura, S. Kera, K. K. Okudaira, Y. Harada and N. Ueno (2001). "Diffusion of chloroaluminum phthalocyanine on MoS2 surface detected by photoemission electron microscopy and metastable electron emission microscopy." J. Appl. Phys. 90(1): 213-216.

${ }^{115}$ H. Yasufuku, M. Okumura, S. Kera, K. K. Okudaira, Y. Harada and N. Ueno (2001). "PEEM and MEEM of chloroaluminum phthalocyanine ultrathin film on $\mathrm{MoS}_{2}$." J. Electro. Spectro. Relat. Phen. 114: 1025-1030.

116 B. O. Leung, A. P. Hitchcock, R. Cornelius, J. L. Brash, A. Scholl and A. Doran (2009). "X-ray Spectromicroscopy Study of Protein Adsorption to a Polystyrene-Polylactide Blend." Biomacromol. 10: 1838-1845.

${ }^{117}$ C. Morin, A. R. Hitchcock, R. M. Cornelius, J. L. Brash, S. G. Urquhart, A. Scholl and A. Doran (2004). "Selective adsorption of protein on polymer surfaces studied by soft X-ray photoemission electron microscopy." J. Electron Spectro. Relat. Phenom. 137: 785-794. 


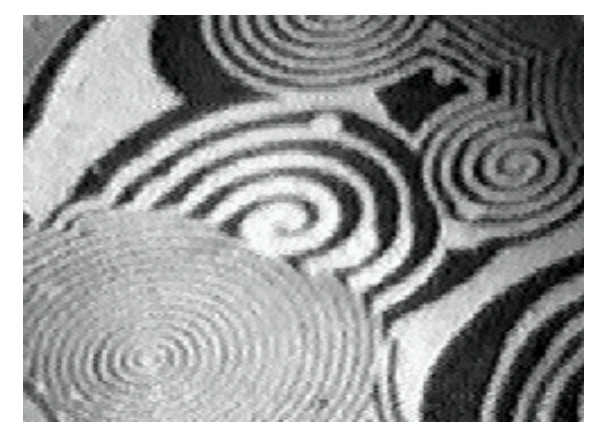

Fig. 1. PEEM image of $\mathrm{CO}$ oxidation on a $\mathrm{Pt}(110)$ surface at $450 \mathrm{~K}$ with $\mathrm{P}_{\mathrm{O} 2}=$ $4 \times 10^{-4}$ mbar and $P_{\mathrm{CO}}=4 \times 10^{-5}$ mbar. Dark and grey regions are oxygen and $\mathrm{CO}$ adsorbed regions, respectively. ${ }^{3}$
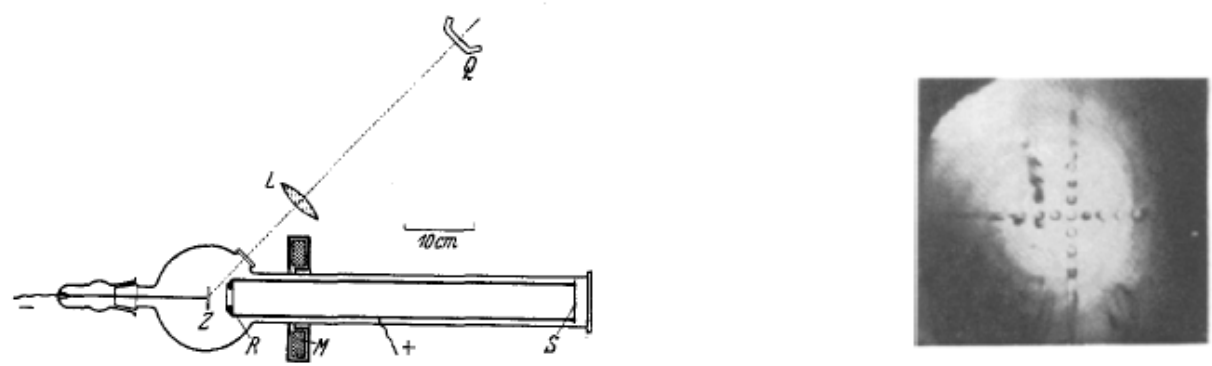

Fig. 2. The PEEM system developed by Bruche and an image obtained for a zinc plate. Q: light source, Z: sample, L: lens, R: objective lens, positively biased (10 kV)

brass tube, M: magnet, S: screen. (Reproduced from Fig.1 and Fig.3 of ref. 16 with kind permission of Springer Verlag) 

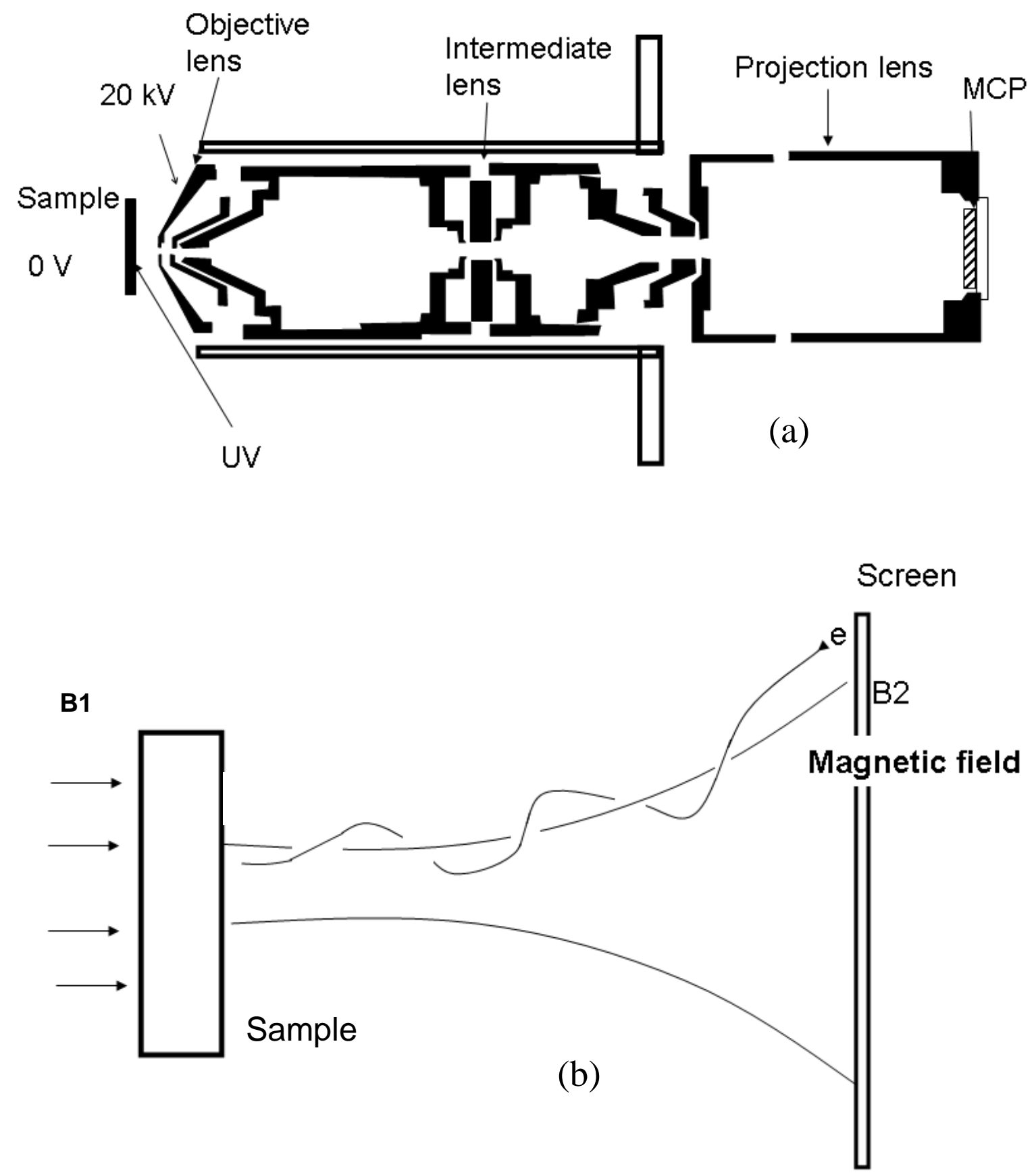

Fig. 3. (a) PEEM $^{3}$ and (b) PESM $^{25}$. In PEEM high voltage is applied between the sample and the objective lens. In PESM the sample is located in a magnetic field. The ejected electron travels around the dispersed magnetic field to provide a magnified image. 
Input lens=

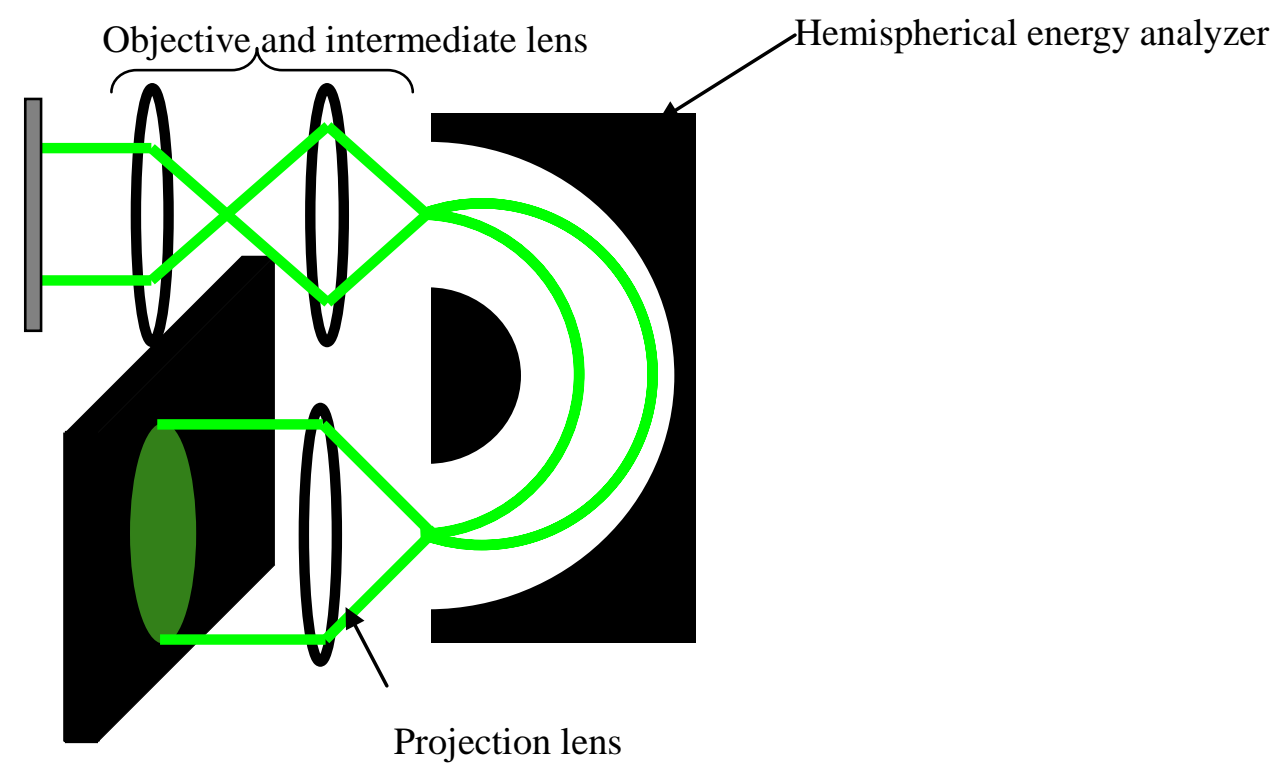

Fig. 4. Schematic illustration of EXPEEM using a hemispherical energy analyzer. PEEM is divided into two parts : One part is input and intermediate lenses and the other is projection lens to magnify and project the surface images. Between them an hemispherical type energy analyzer is inserted to select the photoelectron peaks. 

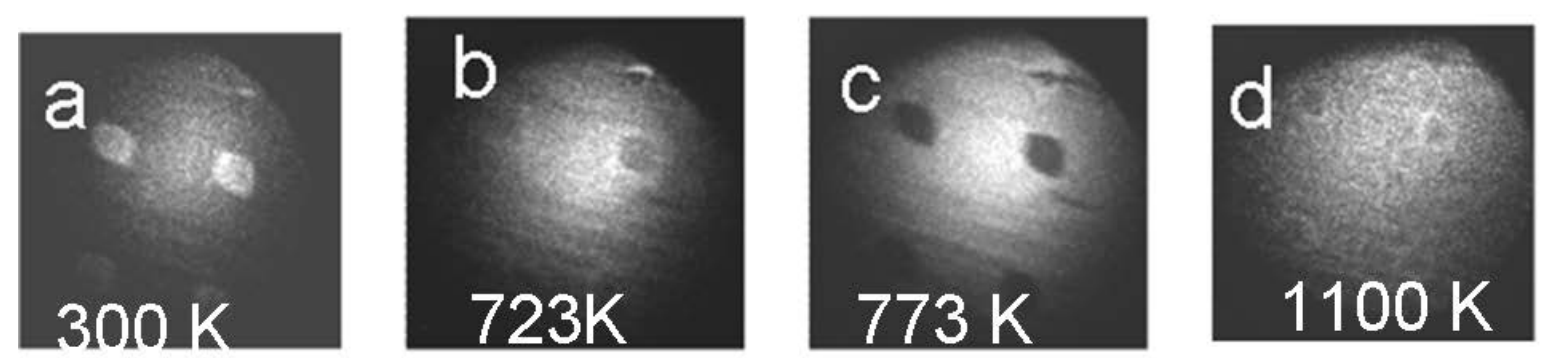

Fig. 5. PEEM images of UV-excited Au islands on a Ta substrate at various temperatures. The

field of view is $80 \mu \mathrm{m}$. 


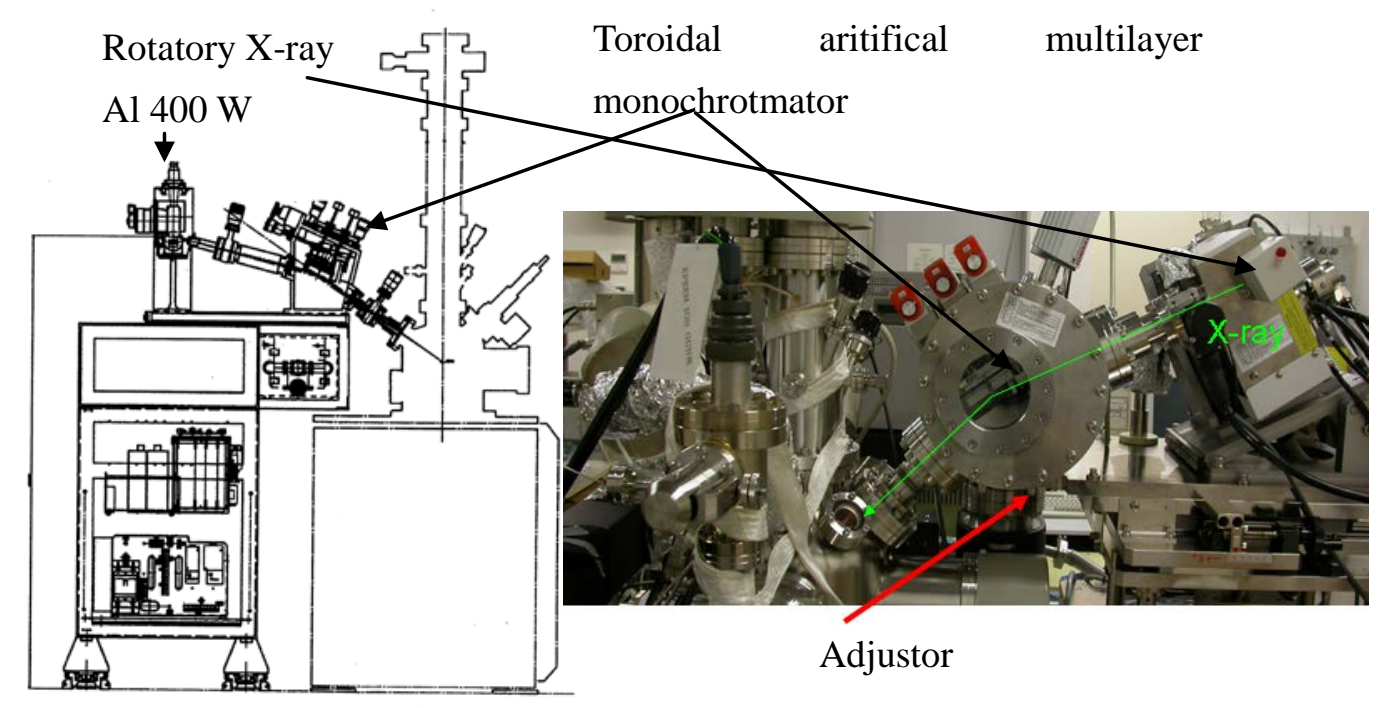

Fig. 6. Rotatory X-ray source with an Al target. X-rays are focused on the sample using a toroidal monochromator
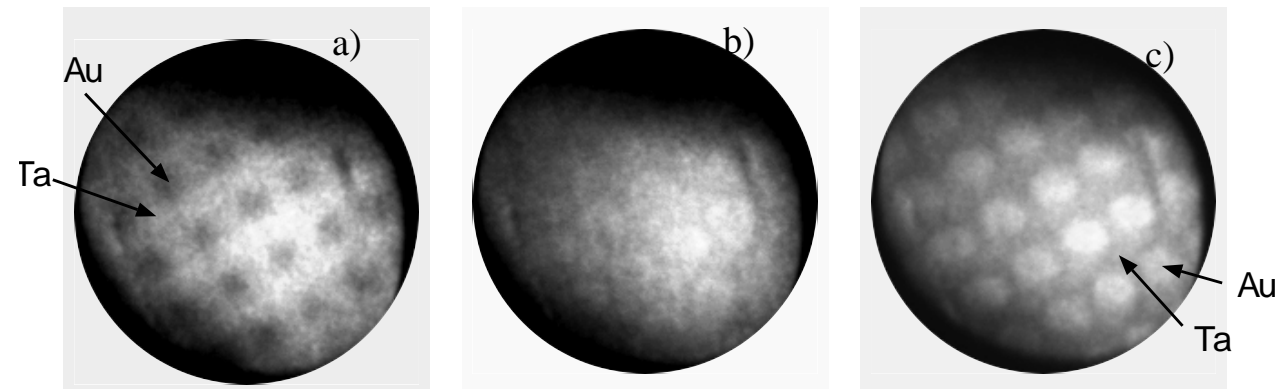

Fig. 7. PEEM image of Au on a Ta substrate excited by synchrotron radiation (Photon factory) a) $h v=\mathbf{2 1 9 5} \mathbf{e V}$, b) $h v=2240 \mathbf{e V}$, and c) $h v=2290 \mathbf{~ e V}$. The $M_{V}$ absorption edge corresponding to the $3 d_{5 / 2}$ state appears at $2240 \mathrm{eV}^{7}$ 

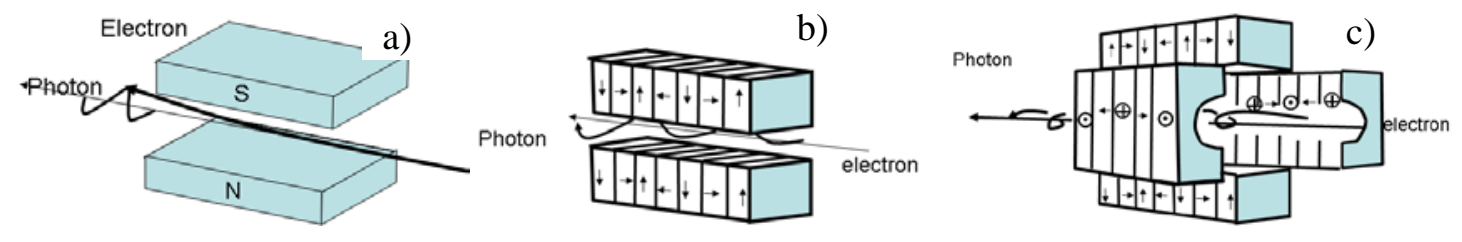

Fig. 8. Photoirradiation from synchrotron sources of (a) a bending magnet, (b) undulator, and (c) helical undulator.

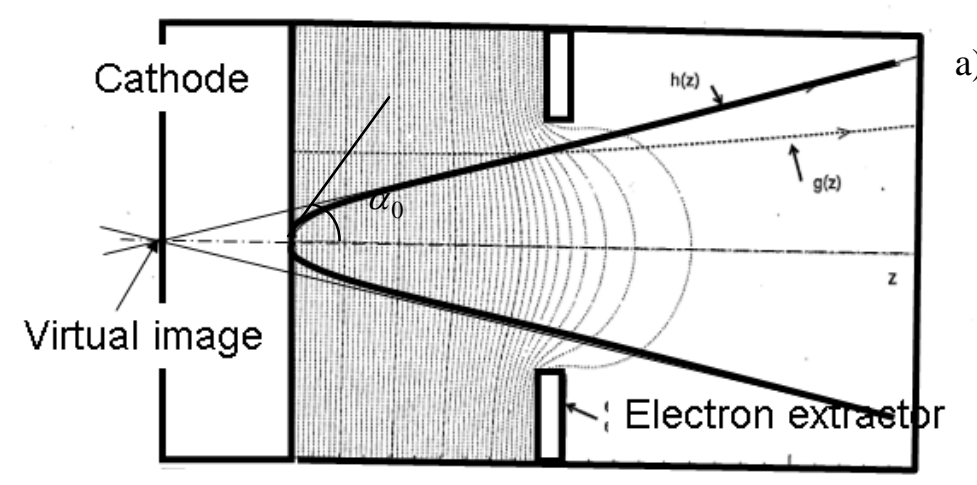

a)

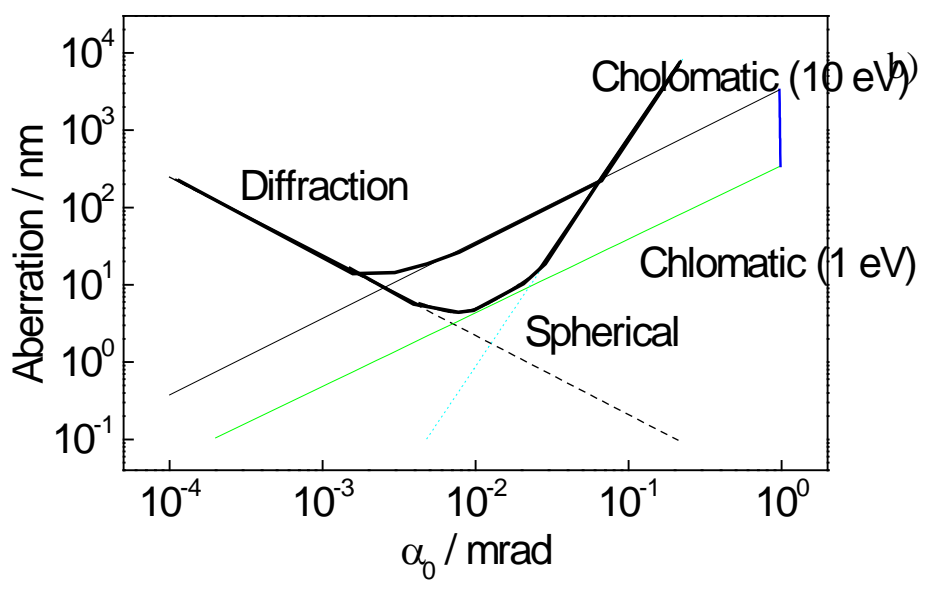

Fig. 9. Principle of the (a) cathode lens, and (b) aberration as a function of $\alpha_{0}$ The sample is a cathode and the extraction electrode is positively charged. A strong electric field is applied between the two electrodes. 


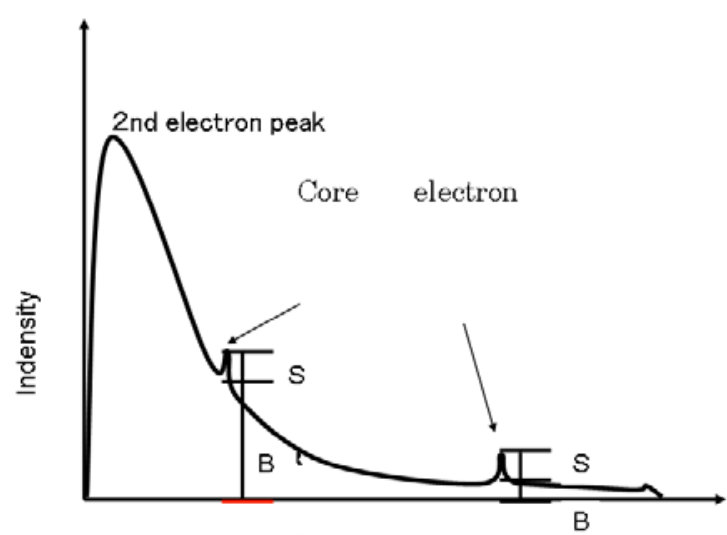

Kinetic energy / eV

Fig. 10. Kinetic energy distribution of photoelectrons excited by high energy $\mathrm{X}$-rays.
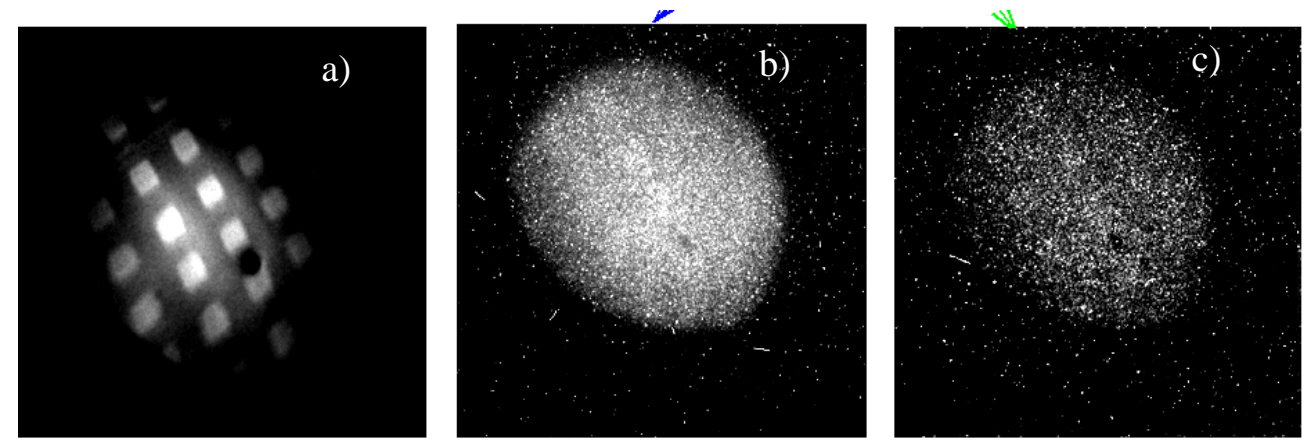

Fig. 11. EXPEEM images of Au island on a Ta substrate obtained using different kinetic energies from excitation by $\mathbf{X}$-rays $(h v=2400 \mathrm{eV})$. (a) $\mathbf{E}_{\mathbf{k}}=\mathbf{0} \mathbf{e V}$, (b) $\mathbf{O} 1 \mathrm{~s}$ peak energy $\left(\mathbf{E}_{\mathbf{B}}=\right.$ $542 \mathrm{eV}$ or $\left.E_{k}=1858 \mathrm{eV}\right)$ photoelectrons and (c) $5 \mathrm{eV}$ higher energy $\left(E_{k} t=1863 \mathrm{eV}\right)$ han the $O 1 \mathrm{~s}$ photo electrons.
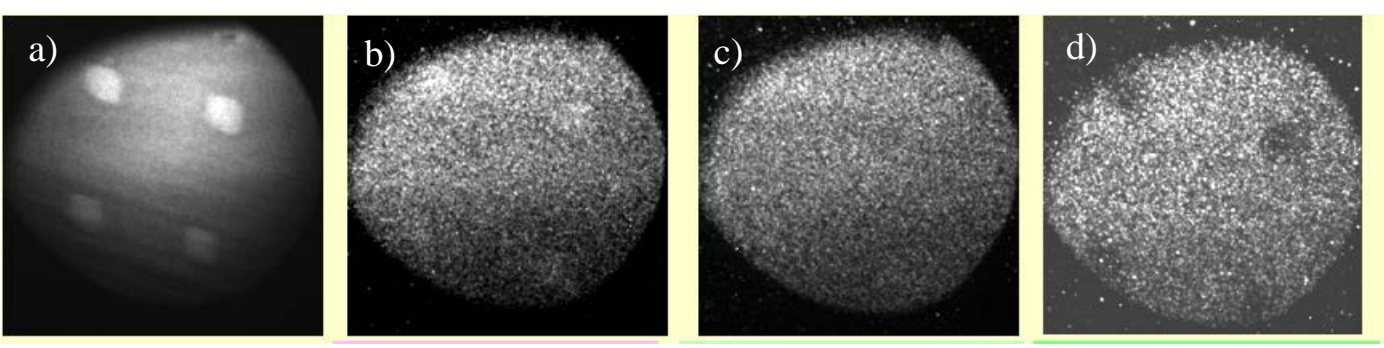

Fig. 12. EXPEEM images of Au islands on a Ta substrate obtained using different kinetic energies. (a) $E_{k}=0 \mathrm{eV}$, (b) $E_{k}=188 \mathrm{eV}\left(\mathrm{Au3d} \mathrm{d}_{5 / 2}\right.$ ), (c) $E_{k}=201 \mathrm{eV}$ (Ta 3p $\mathrm{p}_{3 / 2}$ ), and (d) $E_{k}=653 \mathrm{eV}$

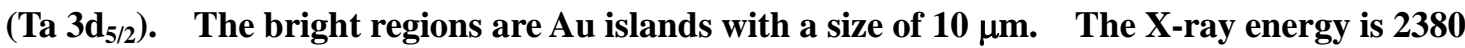
eV. 


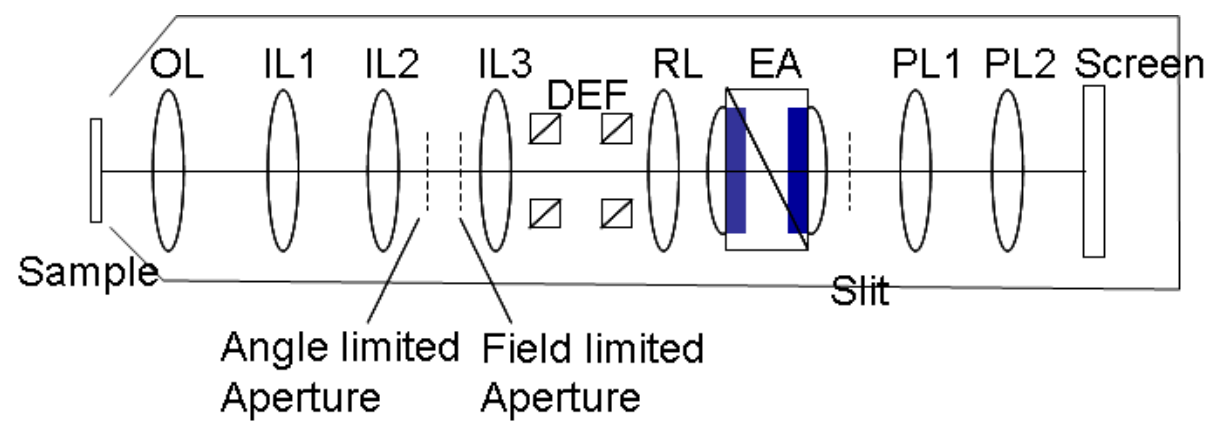

Fig. 13. Schematic diagram of the EXPEEM apparatus. OL: objective lens, IL: intermediate lens, RL: retardation lens, PL1,2: objective lenses, EA: energy analyzer.
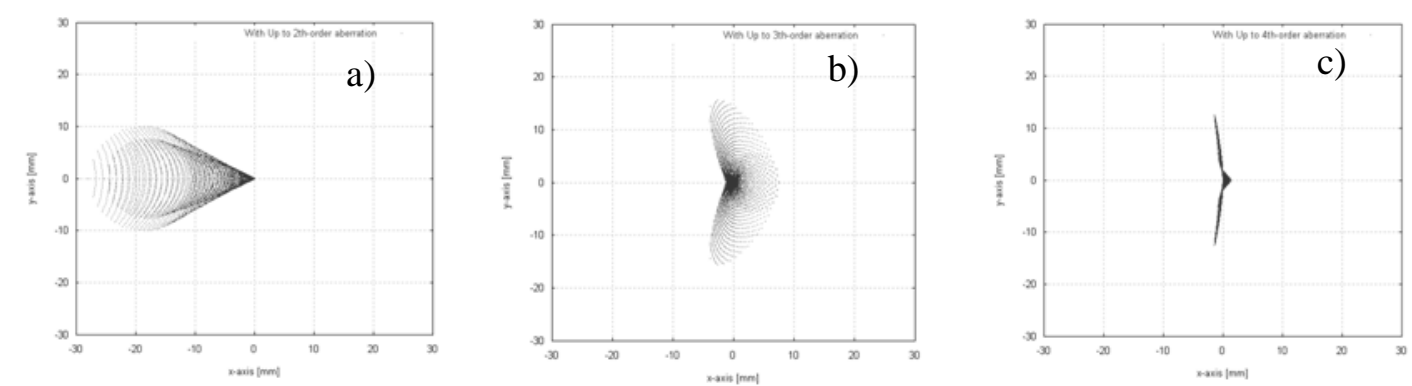

Fig. 14. Aberration figures for aberration reduction conditions. A: stigmatic condition, B: second order aberration correction, $C$ : third order aberration correction. Pass energy of electron $=100$ $\mathrm{eV}$; pass length $=400 \mathrm{~mm}$; maximum of the incident half angle $\left(\alpha_{0 \max }\right)=14^{\circ}$. 

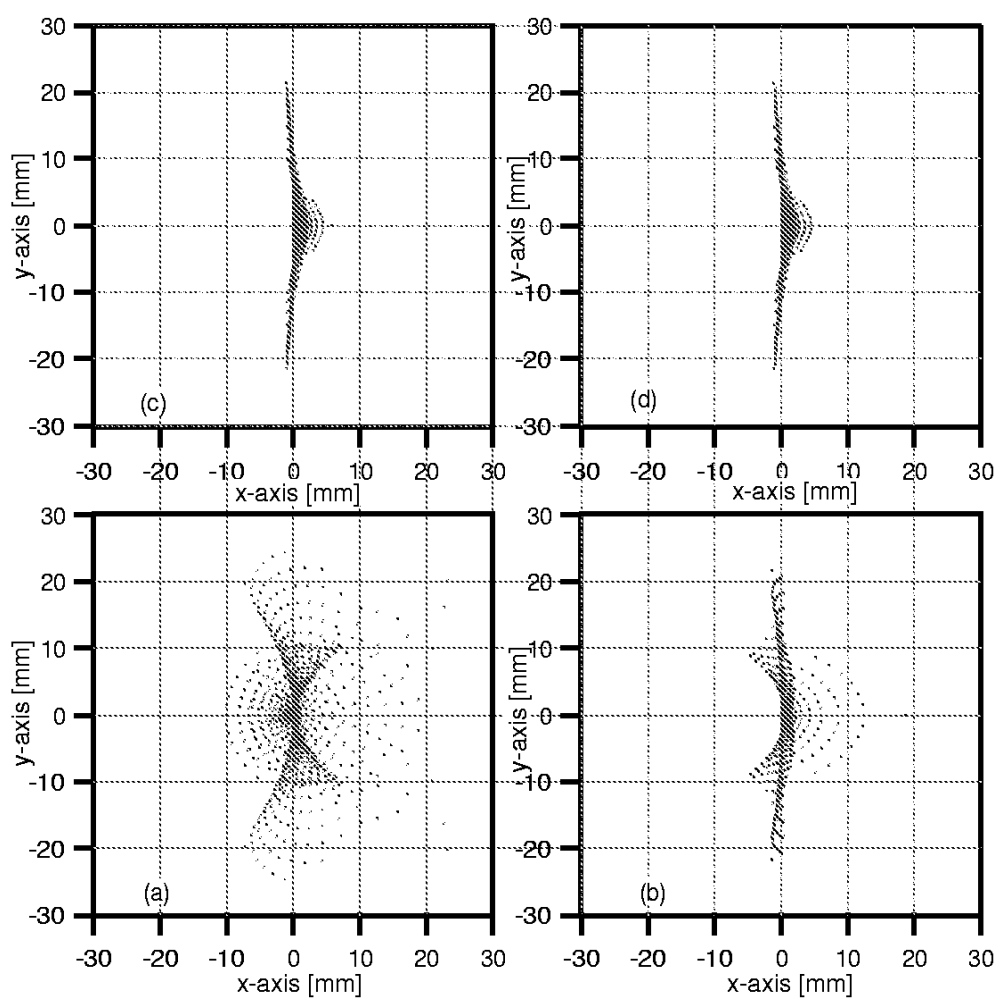

Fig. 15. Aberration figures for (a) 8 electrodes and magnetic poles,

(b) 10 electrodes and magnetic poles, (c) 12 electrodes and magnetic poles, (d)

18 electrodes and magnetic poles. The horizontal direction corresponds to the energy dispersion direction. ${ }^{74}$ 

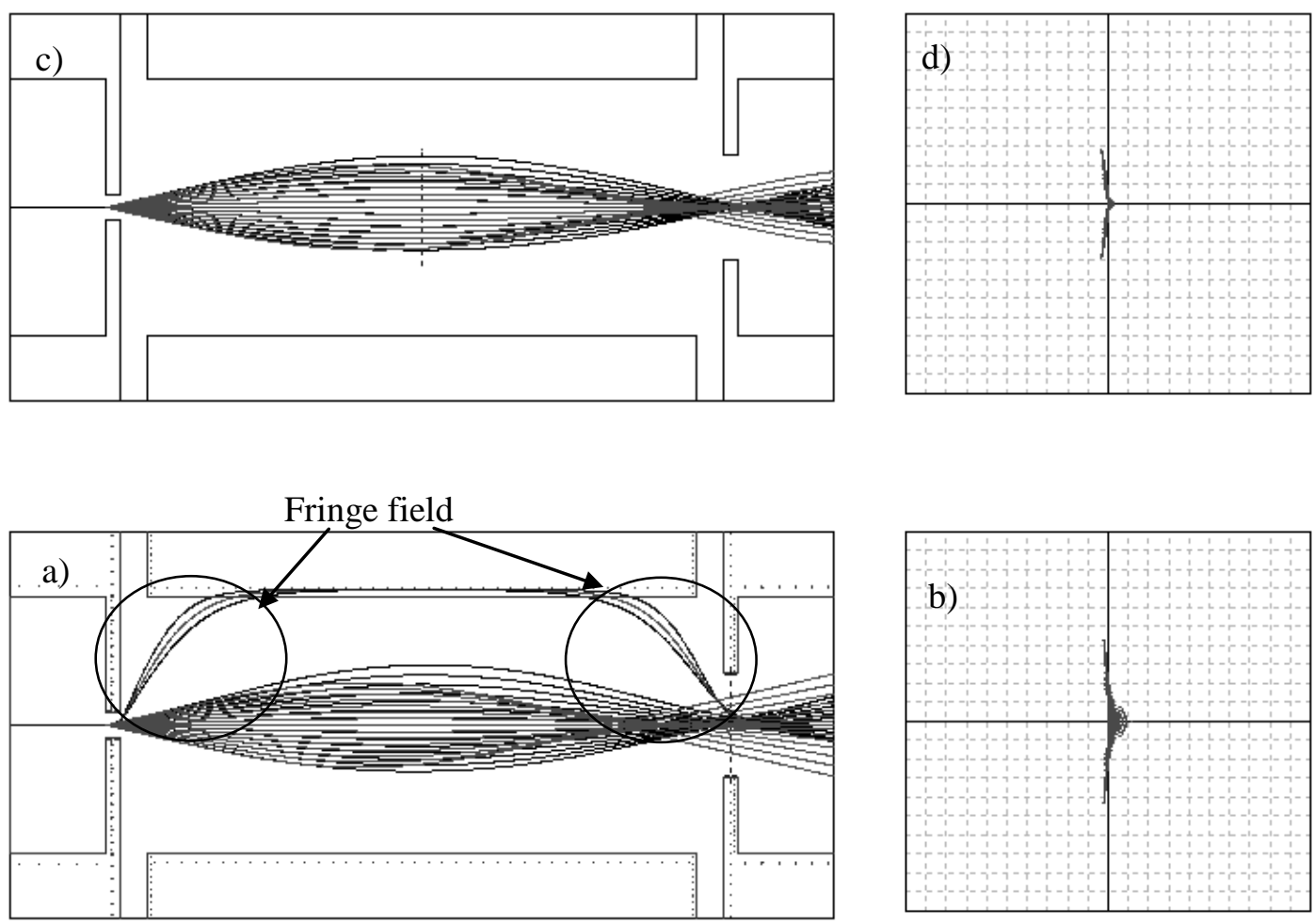

Fig. 16. Electron trajectories $(a, c)$ and aberration figures $(b, d)$. The trajectory and aberration figures are calculated with $(a, b)$ or without $(c, d)$ the fringe field. 


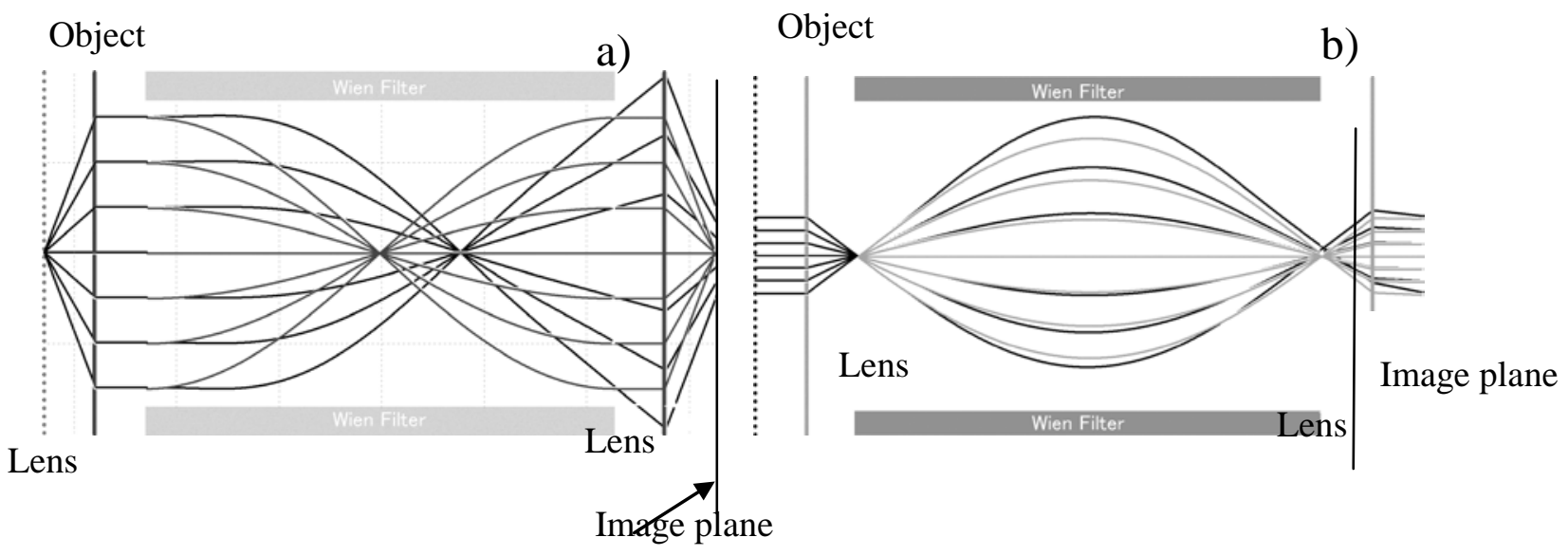

Fig. 17. Two entrance conditions of the Wien filter. (a) A point source and parallel beam enters the Wien filter. (b) A parallel beam is dispersed from the entrance point and enters the Wien filter. Dark lines are the real trajectory, while the thin line is the ideal trajectory without the fringe effect. 

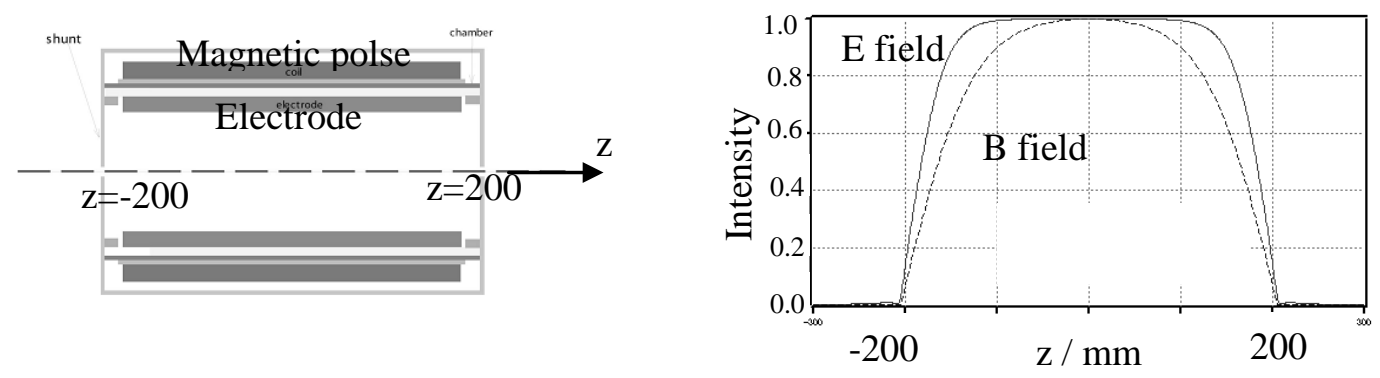

Fig. 18. Wien filter with the same sizes of magnetic poles and electrodes. (a) Setting of the magnetic poles and electrodes, and (b) the corresponding electric and magnetic fields.

E field
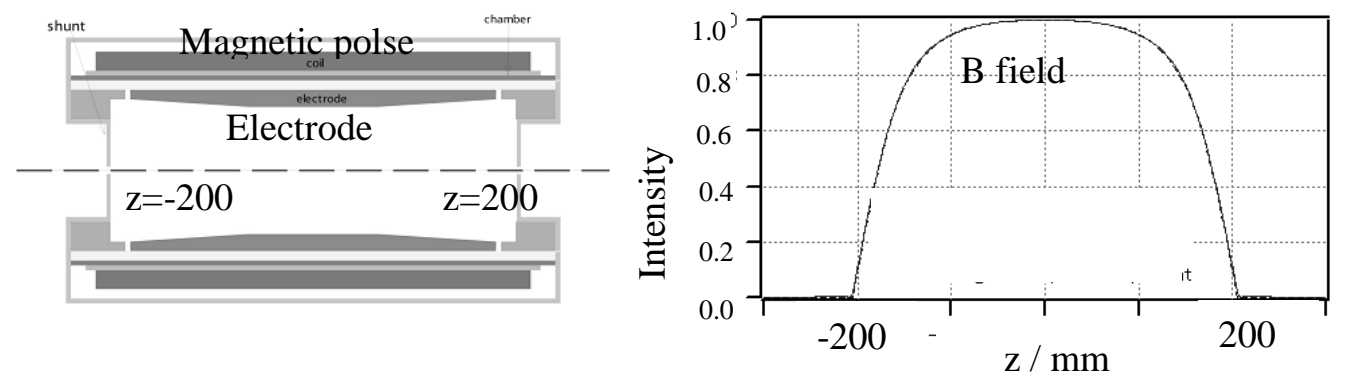

Fig. 19. Wien filter with tapered electrodes and elongated magnetic poles. (a) Setting of the magnetic poles and electrodes, and (b) the corresponding electric and magnetic fields. 
Magnetic polse
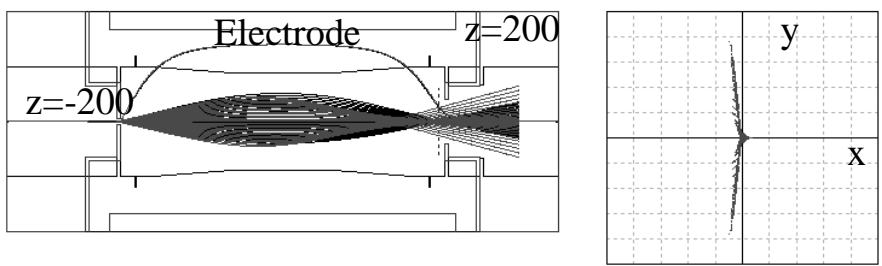

Fig. 20. Trajectory of the tapered electrode Wien filter and the corresponding aberration figure.

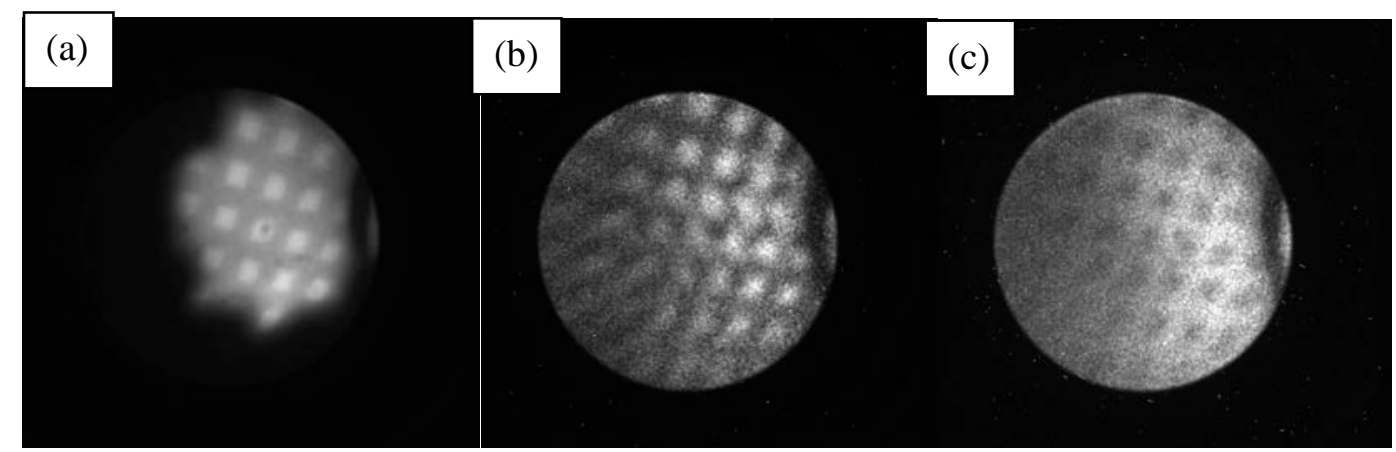

Fig. 21. EXPEEM images of $10 \mu \mathrm{m}$ Au islands on a Ta substrate using (a) $E_{k}=0 \mathrm{eV}$, (b) $A u 4 f_{7 / 2}$, and (c) $\mathrm{Ta} 4 \mathrm{f}_{7 / 2}$.
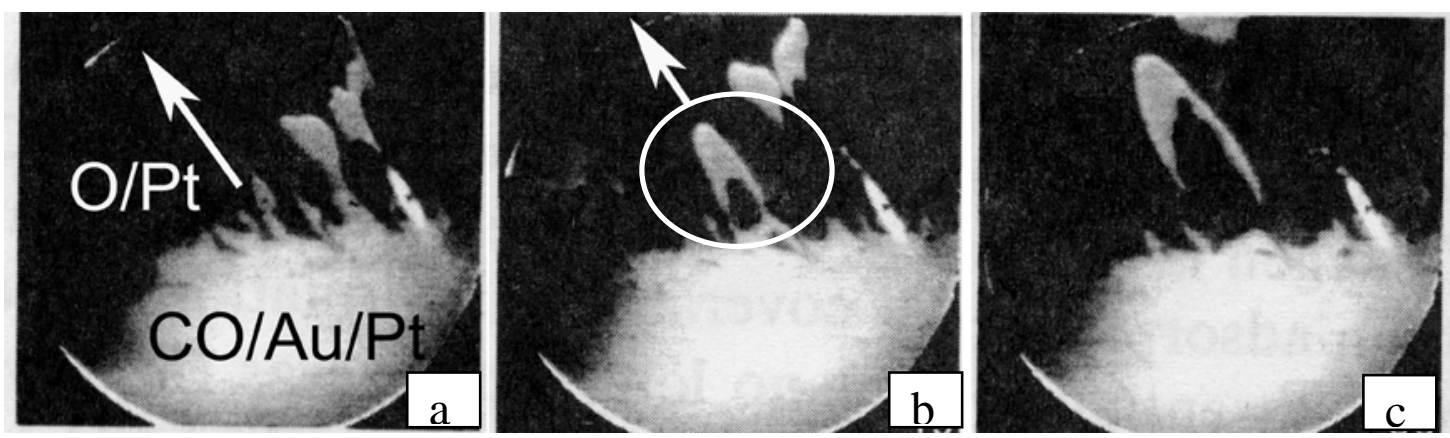

Fig. 22. PEEM images of $\operatorname{Pt}(110)$ partially covered with $0.3 \mathrm{ML}$ Au under CO oxidation reaction conditions. CO: $2.9 \times 10^{-5}$ mbar, $\mathrm{O}_{2}: 4 \times 10^{-4} \mathrm{mbar}$, reaction temperature: $470 \mathrm{~K}$. The $\mathrm{CO}$ covered region appears brighter. $\mathrm{CO}$ is mainly adsorbed on $\mathrm{Au}$-precovered Pt region at the downward bright part. Most of the other part where pure Pt surface is exposed is covered with $\mathrm{O}_{2}$. The $\mathrm{CO}$ concentration wave originating from the Au-precovered region migrates into the $\mathrm{O}_{2}$-adsorbed pure $\mathrm{Pt}$ region. $^{89}$ The interval between images is $4 \mathrm{~s}$. 

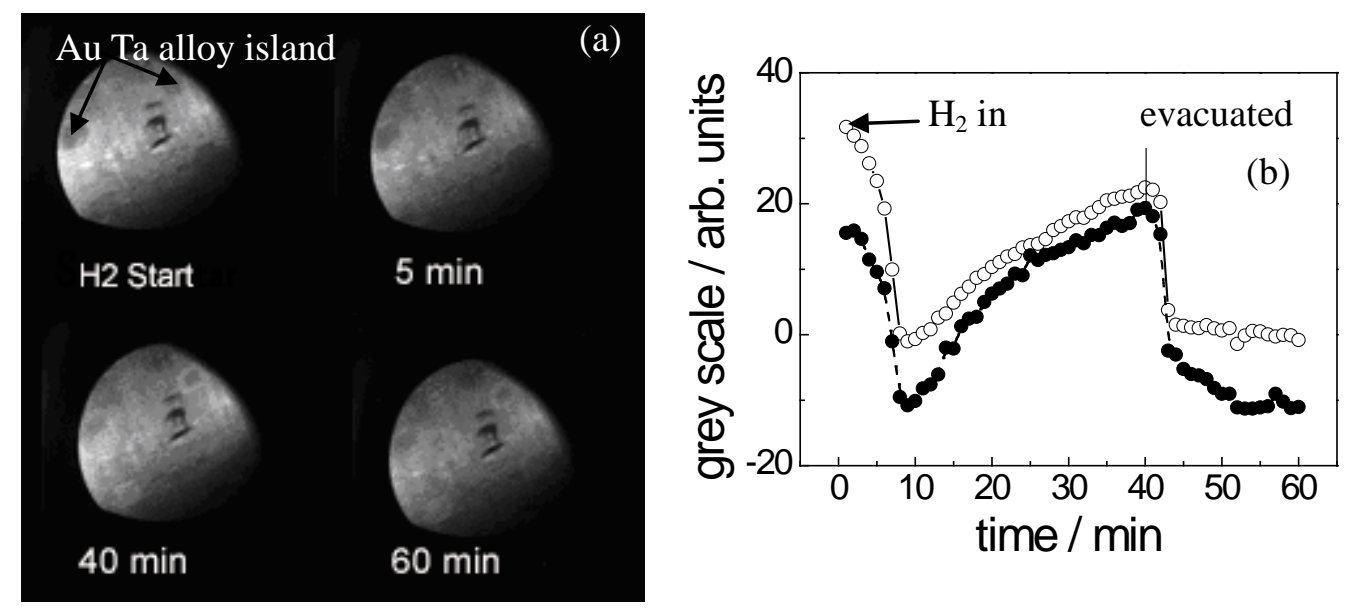

Fig. 23. (a) PEEM images of AuTa alloy islands on the Ta during $\mathrm{H}_{2}$ adsorption. After the introduction of $\mathrm{H}_{2}$, the surface becomes darker and then brighter. After the evacuation, the surface becomes darker again. (b) Contrast change during $\mathrm{H}_{2}$ adsorption and evacuation at 0 $\min$ and $40 \mathrm{~min}$, respectively. ${ }^{32}$

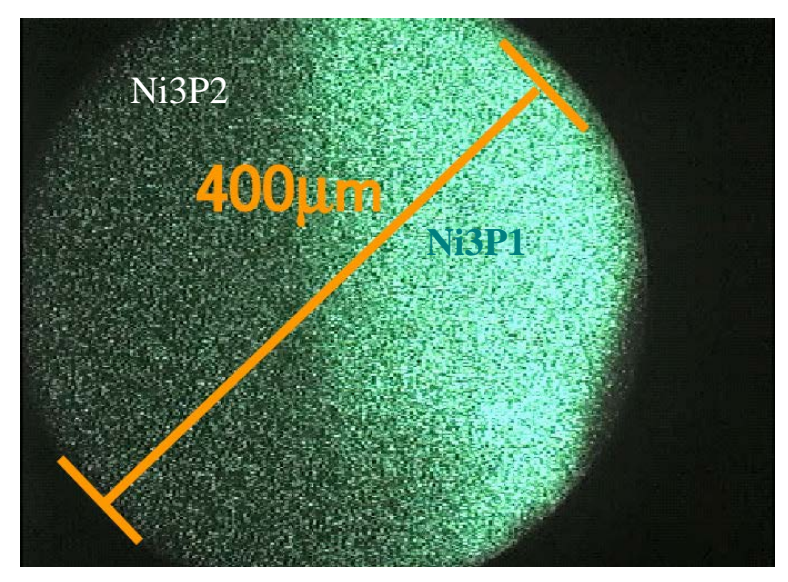

Fig. 24. PEEM image of the $\mathrm{Ni}_{2} \mathrm{P}(0001)$ surface using a UV low path filter. ${ }^{104}$ 


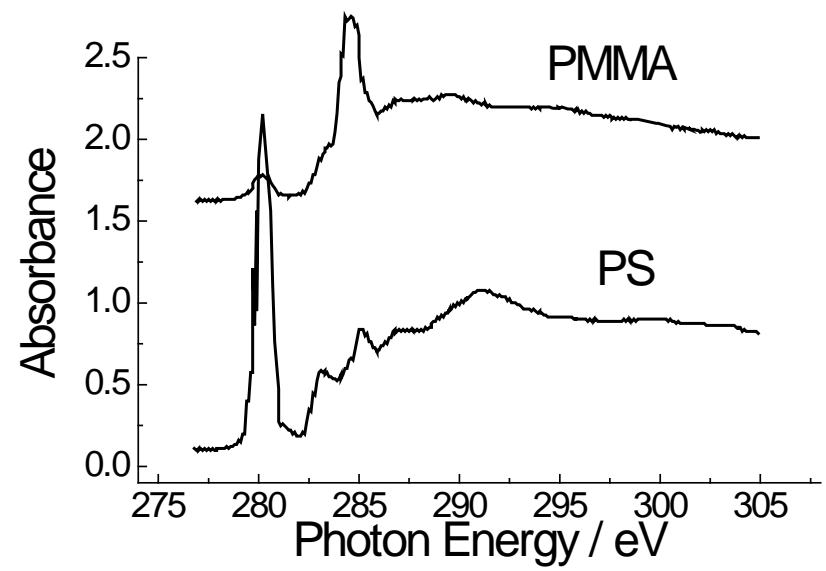

Fig. 25. C 1s NEXAFS spectra of PS and PMMA. ${ }^{112}$ 